\title{
Criminologie
}

\section{La pensée postmoderne et la criminologie (1993)}

\section{Jean-Paul Brodeur et Christian Nadeau}

Volume 44, numéro 1, printemps 2011

Jean-Paul Brodeur, d’hier à aujourd'hui

URI : https://id.erudit.org/iderudit/1001605ar

DOI : https://doi.org/10.7202/1001605ar

Aller au sommaire du numéro

Éditeur(s)

Les Presses de l’Université de Montréal

ISSN

0316-0041 (imprimé)

1492-1367 (numérique)

Découvrir la revue

Citer cet article

Brodeur, J.-P. \& Nadeau, C. (2011). La pensée postmoderne et la criminologie (1993). Criminologie, 44(1), 119-171. https://doi.org/10.7202/1001605ar

\section{Résumé de l'article}

Le présent article est une tentative d'investiguer les diverses significations des mots " postmodernité ", " postmodernisme » et « postmoderne ». Il évalue auss la signification de ces mots et des concepts qu'ils expriment pour la criminologie. L'article est divisé en trois parties. La première tente de dissiper les mésententes importantes qui ont été soulevées par le postmodernisme. Le plus significatif de ces malentendus est la croyance qu'il y a telle chose qu'une méthode postmoderne en sciences sociales. La seconde partie retrace l'origine du terme postmoderne et discute divers thèmes perçus comme caractéristiques de la pensée postmoderne. Ces thèmes sont : la crise actuelle de légitimation, l'analyse du discours et le métalangage, la fragmentation sociale et culturelle, et le pessimisme historique. La dernière partie tire des conclusions des analyses précédentes pour le développement de la criminologie.
Ce document est protégé par la loi sur le droit d'auteur. L'utilisation des services d'Érudit (y compris la reproduction) est assujettie à sa politique d'utilisation que vous pouvez consulter en ligne.

https://apropos.erudit.org/fr/usagers/politique-dutilisation/ 


\section{La pensée postmoderne et la criminologie}

Jean-Paul Brodeur $\left(1993^{\star}\right)$

\section{Note introductive}

Jean-Paul Brodeur fut, comme chacun sait, un immense intellectuel et un très grand chercheur. Pour cette raison, il n'est pas étonnant de retrouver dans la masse des écrits de Brodeur cette longue charge critique contre les abus du postmodernisme. Brodeur présente d'abord les origines historiques de la notion pour ensuite mieux en décrire les thèses récurrentes. Ce n'est que dans la deuxième partie du texte qu'il s'attaque enfin aux influences du postmodernisme sur la recherche en criminologie. Au début des années 1990, une telle démarche peut paraître étonnante, dans la mesure où le postmodernisme et ses protagonistes européens ont connu leurs heures de gloire surtout dans les années 1980, notamment parce qu'il a favorisé la création ou le renouveau de nombreuses disciplines, dont les cultural studies, surtout en Amérique du Nord (longtemps, l'université française a préféré l'œuvre des morts à celle des vivants). En ce sens, il ne faudrait pas non plus jeter le bébé avec l'eau du bain et refuser de reconnaître l'apport immense que fut le postmodernisme, par exemple lorsqu'il s'agit de montrer en quoi un cadre méthodologique d'une science sociale donnée est lui-même orienté en fonction de normes dont les chercheurs se font en quelque sorte les défenseurs involontaires. Mais Brodeur aurait probablement reconnu ce fait, tout en continuant d'expliquer en quoi, dès lors qu'on tente d'en penser les cadres théoriques, le postmodernisme échoue à fournir autre chose qu'une «épistémologie à géométrie

* Criminologie, XXIV (1), 73-121.

Criminologie, vol. $44, \mathrm{n}^{\circ} 1$ (2011) 
variable», dont l'effet pervers est de favoriser l'émergence de normes sociales - comme par exemple en justice pénale - si complexes qu'elles sont vite abandonnées pour laisser place à des transactions entre les parties où règne le droit du plus fort.

Christian Nadeau ${ }^{\star \star}$

RÉSUMÉ • Le présent article est une tentative d'investiguer les diverses significations des mots «postmodernité», "postmodernisme» et "postmoderne». Il évalue aussi la signification de ces mots et des concepts qu'ils expriment pour la criminologie. L'article est divisé en trois parties. La première tente de dissiper les mésententes importantes qui ont été soulevées par le postmodernisme. Le plus significatif de ces malentendus est la croyance qu'il y a telle chose qu'une méthode postmoderne en sciences sociales. La seconde partie retrace l'origine du terme postmoderne et discute divers thèmes perçus comme caractéristiques de la pensée postmoderne. Ces thèmes sont: la crise actuelle de légitimation, l'analyse du discours et le métalangage, la fragmentation sociale et culturelle, et le pessimisme historique. La dernière partie tire des conclusions des analyses précédentes pour le développement de la criminologie.

MOTS-CLÉS - Postmodernité, méthode, pensée postmoderne, analyse du discours, métalangage, fragmentation sociale.

\section{Introduction}

La cote de l'expression «postmodernisme» et de la terminologie qui en est dérivée est en hausse considérable à la bourse du langage. D’abord créée par des auteurs lus par quelques initiés, cette terminologie a essaimé dans les revues savantes et elle envahit aujourd'hui la grande presse, où Madonna est, par exemple, proclamée «la Mae West du postmodernisne» (Dussault, 16 janvier 1993). Les titres d'ouvrages académiques où figurent postmodernisme ou l'un de ses dérivés se sont multipliés de façon accélérée depuis 1990. La fortune du terme étant donc assurée, il faudrait se hâter de déterminer s'il signifie quelque chose de plus précis qu'une façon un peu prétentieuse de désigner «notre époque».

Cet article est consacré à esquisser une première élucidation de l'expression de postmodernisne et de ses dérivés, comme «postmodernité» et «postmoderne». Le texte est divisé en trois parties. La première élabore quelques distinctions préliminaires à un traitement plus systématique de notre sujet et s'efforce de mettre en lumière certaines

\footnotetext{
$\star \star$ Professeur

Département de philosophie, Université de Montréal

christian.nadeau@umontreal.ca
} 
confusions qui existent par rapport à lui. La seconde partie consiste dans un repérage de quelques thèmes communs à la pensée postmoderne. Dans une dernière partie, nous tentons d'évaluer pour la criminologie la signification des thèmes précédemment identifiés.

Il nous importe de souligner d'entrée de jeu que notre entreprise est de nature résolument exploratoire et que nous avons pleinement conscience, en outre, que notre terrain d'exploration est parsemé d'embûches. Selon l'article approfondi de Welsch (1988: 9-11) portant sur la généalogie du terme "postmoderne», cette expression est controversée d'au moins quatre manières: on conteste autant sa légitimité, son champ d'application, qui s'étend maintenant de la théologie à la gastronomie et au tourisme, que son apparition dans le temps et son contenu. Le caractère polémique du débat sur le postmodernisme est très marqué, comme en témoignent des ouvrages qui présentent ce débat comme un champ de bataille (Ruby, 1990) ou un combat pour l'avenir de la pensée (Kemper, 1988); d'autres ouvrages s'autorisent des excès de langage - Kroker et Cocke (1986) qualifient la «scène postmoderne» de «culture excrémentielle» (excremental culture) - qui témoignent également de la vivacité de la controverse sur le postmodernisme.

Un exemple est apte à illustrer de façon explicite les difficultés que nous devrons affronter. La revue américaine Telos a publié en 1983 une interview de Michel Foucault par le philosophe Gérard Raulet. Dans l'une de ses questions, Raulet demande à Michel Foucault comment il se situe par rapport au concept de postmodernité, dans lequel lui, Raulet, voit un pôle autour duquel se cristallisent un grand nombre des thèmes de la pensée actuelle. Affirmant de façon faussement naïve que ses informations ne sont pas à jour, Foucault demande à son interviewer de l'éclairer sur le sens de l'expression «postmodernité». Ce dernier fait alors remarquer à Foucault que dans un des derniers textes d'Habermas, où il prend à partie le courant postmoderne, celui-ci attribue la paternité de ce courant à une tradition française de pensée, qui s'ouvre avec Georges Bataille et conduit à Jacques Derrida à travers nul autre que lui-même, Michel Foucault. Il s'ensuit un échange assez compassé entre Michel Foucault et son interlocuteur (voir Foucault et Raulet, 1983 : 204-205; pour la position de Habermas sur Foucault, voir Habermas 1985 et 1989c).

Nous reviendrons plus tard sur le contenu de l'entretien entre Michel Foucault et Gérard Raulet. Il suffira pour l'instant de remarquer le caractère enchevêtré d'une conjoncture de pensée où l'on attribue une 
paternité à un auteur qui se déclare ignorant de sa progéniture. C'est donc à pas mesurés que nous devrons nous aventurer sur ce terrain miné. Dans le cadre trop restreint de cet article, notre ambition est plus d'identifier les questions que soulève la problématique du mot «postmoderne» que de les résoudre.

\section{Postmodernisme, postmodernité et postmoderne}

Rose (1991) constitue l'ouvrage de référence pour l'histoire de la terminologie du mot «postmoderne» et de ses dérivés. Dans sa conclusion, Rose (1991: 171 et ss) distingue 38 usages originaux du mot «postmoderne» et des autres termes formés à partir de cet adjectif. Dans trois de ces usages, le terme sert à des historiens pour désigner une période de l'histoire. Dans 9 autres cas, cette terminologie est utilisée pour dénoter des concepts empruntés à la philosophie ou aux sciences sociales. Dans les 26 autres usages, la terminologie du mot «postmoderne» renvoie à diverses écoles dans le domaine de la poésie et de la littérature (7 usages) et surtout dans celui des beaux-arts (19 occurrences). En conformité avec cette analyse, nous répartirons les différentes façons de combiner le préfixe post avec le terme «moderne» et ses dérivés en trois classes, à partir desquelles nous estimons opportun de distinguer trois objets.

(i) Le premier est une esthétique ou une certaine conception du beau qui est surtout appliquée aux champs des arts visuels et de façon plus particulière à l'architecture et à la peinture (Jencks, 1987; Portoghesi, 1983). On peut également rattacher à ce courant, qui se manifeste surtout dans les arts, une certaine pratique de la critique littéraire, bien que celle-ci déborde le cadre d'une esthétique pour se produire comme une théorie de la littérature (Hassan, 1971, 1975, 1985 et 1987). Nous réserverons à ce premier courant qui consiste en une esthétique des arts visuels et une théorie de la littérature l'appellation de postmodernisme. Nous n'en traiterons pas dans le cadre de cet article, sinon pour dissiper le malentendu que peut faire naître le postmodernisme ainsi défini.

(ii) Le second des objets qu'il importe de distinguer est une époque de l'histoire de l'Occident. Nous utiliserons l'expression «postmodernité» pour nous référer à cette époque, dont les limites sont déterminées de façon variable selon les auteurs. Il importe de souligner que, dans la terminologie que nous proposons, la postmodernité ne désigne pas une approche ou une perspective théorique; elle consti- 
tue le référent extra-linguistique d'une telle approche ou d'une telle perspective, à savoir une période historique.

(iii) Enfin, nous userons de l'adjectif «postmoderne» de deux façons. Nous l'utiliserons d'abord pour qualifier un courant de pensée théorique, surtout issu de la philosophie, qui propose une interprétation de certains aspects de la conjoncture historique dans laquelle nous nous trouvons. Nous l'utiliserons ensuite pour caractériser l'interprétation elle-même de notre conjoncture historique proposée par le courant auquel nous venons de référer. Selon le premier usage de l'adjectif "postmoderne», on parlera de la pensée postmoderne et, selon le second, de la condition postmoderne. Dans le premier cas, l'adjectif «postmoderne» est appliqué à la source d'une interprétation - la pensée postmoderne - et dans le second cas, il est appliqué au produit du processus d'interprétation, à savoir la condition postmoderne, qui est donc un objet construit. Nous pourrons à l'occasion nous référer à l'une ou l'autre de ces réalités - la pensée et la condition postmoderne - ou aux deux prises ensemble par la forme substantivée le «postmoderne».

Notre but en proposant ces distinctions n'est pas de normaliser l'usage d'un vocabulaire mais d'éviter que ne soient confondus des aspects très différents de notre champ d'investigation. Ce champ est en effet constitué par une esthétique, par une réalité historique conçue comme un âge nouveau et par une réflexion théorique qui prend cette réalité pour son objet et qui en produit une interprétation déterminée.

Nous allons maintenant reprendre chacun de ces aspects de notre thème de recherche et tenter de conjurer les nombreux malentendus auxquels ils peuvent donner naissance.

\subsection{Le postmodernisme en tant qu'esthétique}

Une esthétique est essentiellement un discours normatif. Elle présente une conception idéale du beau ou encore de l'œuvre d'art réussie, et elle prescrit les règles à suivre pour parvenir à cet idéal. Elle s'apparente à cet égard à une éthique, qui édicte les règles de la vertu et du bien moral, et elle se rapproche également d'une méthode ou d'une logique, lesquelles fixent les canons de la vérité et formulent les procédures qui doivent être suivies pour produire des énoncés théoriques vrais.

Cet aspect régulateur de l'esthétique se manifeste pleinement dans les travaux précités de Jencks, de Portoghesi et même dans la nouvelle 
critique littéraire qui exalte l'œuvre de certains écrivains présentés comme des modèles (Joyce, Proust, Borges, etc.). Le caractère propre de cette esthétique est d'être suffisamment constituée pour avoir donné naissance à un corps de doctrine et à des œuvres qui la représentent, la nourrissent et lui permettent d'évoluer.

L'aspect développé du postmodernisme en tant qu'esthétique a pu engendrer la croyance qu'il était parvenu à un même degré d'explicitation dans le domaine de l'épistémologie et de la méthodologie des sciences sociales. Cette croyance constitue en réalité une illusion profondément trompeuse. Il existe à n'en pas douter des ouvrages théoriques qui interrogent certains aspects de la postmodernité en dehors du champ de l'esthétique. On peut mentionner, parmi beaucoup d'autres, les travaux d'Anthony Giddens $(1990,1991)$ en sociologie, ceux de Robert Reiner (1993) en criminologie et ceux de Jean-François Lyotard (1979) qui se sont penchés sur les traits du savoir dans la conjoncture de cette fin de siècle. Il n'existe toutefois pas de traité de méthodologie ou d'épistémologie énonçant les principes du postmodernisme de la même façon que furent élaborés les principes du structuralisme dans diverses disciplines des sciences sociales, comme au premier chef la linguistique et l'anthropologie.

Cette illusion selon laquelle il existerait quelque chose qui ressemblerait à une méthodologie du postmodernisme repose pour l'essentiel sur une projection dans les domaines des sciences sociales d'un degré d'explicitation qui n'existe que dans le champ de l'esthétique. Cette projection a été facilitée par le fait que plusieurs des critiques du postmodernisme ont délibérément confondu ses dimensions esthétiques et théoriques. Le sociologue américain Daniel Bell est peut-être le premier chercheur à avoir lancé, dès 1976, la charge contre le postmodernisme. Or, Bell (1976: 34 et 51-52) définit le postmodernisme comme un tempérament (temper) attribué à des gens aussi différents que des chercheurs comme Norman O. Brown et Michel Foucault, le psychanalyste R. D. Laing et les écrivains Jean Genet, William Burroughs, auteur de Naked Lunch (Le festin nu), et Norman Mailer. De façon similaire, dans sa critique marxiste du postmodernisme, Alex Callinicos (1989) s'en prend d'abord contre une esthétique; il critiquera par la suite des penseurs comme Foucault et Derrida, qui se sont souvent opposés dans leurs écrits et qui ne sont pas qualifiés de postmodernistes par Callinicos, mais plutôt de poststructuralistes. 
Il faut en outre insister sur le fait que Lyotard, l'un des principaux protagonistes du débat sur le postmoderne, a produit des travaux autant dans le domaine d'une théorie du savoir (Lyotard, 1979) que dans celui d'une esthétique de la peinture (Lyotard, 1980, 1982, 1983). Lorsque Lyotard (1988: 28-32) tente d'apporter une réponse à la question «qu'est-ce que le postmoderne?», il formule cette réponse essentiellement dans le champ d'une esthétique picturale.

Il est toutefois d'autres facteurs qui nourrissent l'illusion d'une méthodologie postmoderne. Dans une mesure non négligeable, le postmoderne est une construction de la critique qui regroupe sous une même bannière des auteurs qui s'étonnent de marcher sous le même drapeau; dans Der philosophische Diskurs des Moderne (Le discours philosophique de la modernité), ouvrage phare, qui a véritablement lancé la controverse sur la pensée postmoderne, bien que ce terme n'y apparaisse pas, Habermas (1985) critique des auteurs aussi différents que Hegel, Nietzsche, Horkheimer, Adorno, Heidegger, Derrida, Bataille et Foucault. Les travaux qui utilisent la terminologie du postmoderne ayant suscité des réactions de plus en plus vives, on est porté à mesurer à partir de la vivacité d'une critique le degré de réalité de son objet. Il est toutefois un dernier facteur qui alimente d'une façon déterminante l'illusion d'une méthodologie du postmoderne et dont nous traiterons dans la section 1.3 de cet article.

\subsection{La postmodernité comme réalité historique}

L'expression de réalité historique que nous utilisons comporte une double signification. Elle signifie d'abord que la postmodernité est le corrélat externe d'une interprétation; en d'autres termes, la postmodernité n'est pas un discours mais l'objet d'un discours. Cet objet comporte toutes les dimensions que l'on prête à la réalité humaine et il peut être conçu (i) comme un état de la société et de ses institutions, (ii) comme un état de la conjoncture politique, (iii) comme un état de la culture et de la production artistique ou enfin (iv) comme un état du savoir. Le qualificatif «historique» signifie que la postmodernité est un objet situé dans le temps, ce qui est explicitement inscrit dans la désignation de postmodernité. Ces deux aspects de la postmodernité, ses aspects objectal et temporel, ne sont pas également sans soulever quelques difficultés, qui naissent du jeu des relations entre ces deux aspects. 
A. Une première difficulté tient aux variations que l'on peut constater dans la détermination de la période de l'histoire que recouvre la postmodernité. En effet, selon l'aspect de la postmodernité retenu, on marque le commencement de cette période de façon profondément différente. Abordée sous le biais de l'architecture, la postmodernité ne remonte guère au-delà des années soixante, si l'on excepte les réalisations de certains architectes hors catégories comme le Catalan Gaudi. Pour un historien de la philosophie de stricte obédience, la postmodernité pourrait être conçue comme l'époque qui succède à la philosophie moderne représentée par Descartes et les grands cartésiens (Malebranche, Spinoza et Leibniz). Dans cette perspective, la postmodernité coïnciderait avec l'époque dite des Lumières, à savoir le XviII ${ }^{\mathrm{e}}$ siècle européen. Pour d'autres interprètes, plus centrés sur les phénomènes sociaux, c'est l'Âge moderne qui débute avec les Lumières et la postmodernité ne peut donc commencer qu'après l'effondrement - difficile à dater de façon précise - de la structure de société élaborée à partir des idéaux de la Révolution française. Le premier historien à avoir utilisé l'expression «postmoderne» est Arnold Toynbee. Dans son usage initial, qui date de 1939, Toynbee applique le qualificatif de postmoderne à l'époque qui débute avec le premier conflit mondial (1914); à partir de 1946 et de façon plus particulière dans un ouvrage paru en 1954, Toynbee fait débuter l'Âge postmoderne en 1875 (Rose, 1991 : 171-172). Ces variations ne constituent pas un obstacle décisif à l'utilisation du concept de postmodernité en histoire, à la condition d'être averti des aspects de la postmodernité auxquels le concept est rapporté par un auteur.

B. L'adjectif «moderne» vient de l'adverbe latin «modo», qui signifie «récemment». D'où la difficulté d'avoir une compréhension intuitive d'un concept selon lequel il y a une époque qui vient après celle qui est la plus récente (la plus «moderne»). On trouve une difficulté logique équivalente à tenter de concevoir «le bout d'une extrémité». Cette difficulté se résout en soulignant que l'interprétation bistorique de l'Âge moderne a supplanté son interprétation lexicale ou sémantique. L'Âge moderne désigne cette période de l'histoire de l'Occident qui a succédé à celle du Moyen-Âge. Dans les manuels d'histoire, l'Âge moderne s'achève en 1789 , avec la Révolution française et est suivie de l'époque contemporaine. Pour les auteurs que nous commentons, le modernisme s'étend bien au-delà de la Révolution 
française. Dans tous les cas, il n'y a pas d'aporie à faire suivre le moderne par le postmoderne, pourvu que l'on adopte pour la signification du mot «moderne» une perspective historique plutôt que sémantique.

C. Nous avons déjà dit que la postmodernité désignait un objet de pensée ou, pour parler plus précisément, qu'elle constituait le corrélat d'une interprétation. Nous voulions marquer par cette dernière expression que la postmodernité, en vertu même de sa désignation de postmodernité, était le produit de présuppositions théoriques beaucoup plus structurantes que si cette époque n'était désignée simplement que par sa place dans le temps (par exemple, «le $\mathrm{xx}^{\mathrm{e}}$ siècle» ou «la période qui s'étend de la Révolution russe jusqu'à aujourd'hui»). Le présupposé théorique déterminant par rapport à la postmodernité est que cette époque marque une rupture avec celle qui la précède. Il faut en effet se distinguer de façon radicale d'une époque pour prétendre lui succéder. Or, ce présupposé fait maintenant l'objet d'une contestation systématique de la part du sociologue britannique Anthony Giddens. Pour Giddens, bien loin de rompre avec les temps qui la précèdent, l'époque actuelle est au contraire l'exacerbation des tendances de la modernité. C'est pourquoi il désigne notre époque par le thème «haute modernité » (bigh modernity) ou «modernité radicalisée» (radicalized modernity). Giddens (1990: 150) nous fournit, sous la forme d'un tableau, les points d'opposition entre sa conception de la haute modernité et celle des théoriciens de la postmodernité, représentés au premier chef par Lyotard. Nous nous abstiendrons de prendre partie dans ce débat, que nous n'utilisons que pour appuyer notre assertion que la postmodernité est le corrélat d'une interprétation, c'est-à-dire un objet qui est ab initio le produit d'une construction. En effet, Giddens est loin d'être le seul chercheur qui conçoive le postmoderne comme le produit d'une logique de la modernité poussée jusqu'à la démesure; Bell (1976), Habermas (1985) et Callinicos (1989), lorsque ce dernier se réfère à des «poststructuralistes» comme Foucault ou Derrida, partagent cette conception. 


\subsection{Le postmoderne comme résultat conceptuel d'un travail théorique de mise en forme}

Le postmoderne est un objet doublement construit. Sous les traits de la postmodernité, il reçoit une première mise en forme par l'opération de présupposés impliquant que cette période est en rupture avec la précédente, ne serait-ce au sens minimal qu'elle lui succède et inaugure une ère nouvelle. Cette première construction est cependant purement formelle et abstraite: elle se borne à affirmer que les traits de la postmodernité sont dans un rapport d'opposition différentielle avec ceux de la modernité. La nature de ces traits n'est toutefois pas explicitée. Cette explicitation ne pourra être produite que par un travail théorique élaboré. Son résultat est le postmoderne, qui est donc le fruit d'une réflexion sur les caractères de la postmodernité.

Nous allons maintenant reprendre une question que nous avons laissée en suspens à la fin de la section 1.1. Nous tentions alors d'identifier quels étaient les facteurs qui favorisaient la genèse de l'illusion que le postmoderne était une méthode, ou mieux, une épistémologie. On peut formuler à nouveau cette question dans les termes de la discussion présente. Le postmoderne, affirmons-nous, est un objet doublement construit. On pourrait également se demander si cet objet doublement construit peut fournir le modèle d'un processus de construction d'autres objets ou si, en d'autres termes, les traces de sa construction sont suffisamment marquées pour qu'on tente de les scruter afin de reconstituer la méthode du constructeur.

Cette question est complexe et nous ne pouvons en traiter en évitant toute technicité. Nous avons déjà vu que cette réalité historique que constituait la postmodernité comportait plusieurs aspects. Par l'un de ses aspects, la postmodernité est un état du savoir et de l'expression de la pensée théorique. Le penseur qui réfléchit sur cet aspect de la postmodernité se penche en conséquence sur des expressions de la pensée théorique. Autrement dit, ce sont des discours (théoriques) qui constituent l'objet de ses analyses, qu'il exprime dans un métalangage (un langage de second degré qui porte sur des langages visés en objet). Or, par un processus dont nous tenterons de rendre compte, il arrive fréquemment que les énoncés d'un métalangage sont reportés sur lui-même et son auteur. Par exemple, lorsque Bauman (1987) propose la thèse que les intellectuels de la modernité exercent une fonction de législateurs, alors que ceux de la postmodernité agissent plutôt comme des inter- 
prètes, nous sommes naturellement portés à penser que Bauman, qui est notre contemporain, exerce lui-même une fonction d'interprète et qu'il nous convie à suivre son exemple et celui des intellectuels de la postmodernité.

Pourquoi sommes-nous entraînés à penser ainsi? Parce que nous sommes pris au jeu de la réflexivité interne de certains énoncés, selon laquelle une proposition est mise en cause par son contenu. L'exemple achevé de la réflexivité interne des propositions est fourni par le paradoxe du menteur, qu'on peut formuler ainsi:

Épiménide le Crétois dit que les Crétois sont des menteurs.

Si Épiménide dit vrai, il est en conséquence faux que les Crétois sont des menteurs, car Épiménide est lui-même un Crétois et dans cette mesure il est lui-même un menteur et parle en faux des Crétois. À partir de ce premier rabattement sur Épiménide de son propre propos, on peut engendrer une série indéfinie de paradoxes, chaque affirmation donnant naissance à son contraire.

De façon analogue, on peut construire un ensemble de propositions qui montrent que les exégètes du postmoderne sont captés par la réflexivité interne de leurs énoncés. Par exemple:

Bauman, l'intellectuel postmoderne, affirme que les intellectuels postmodernes sont plutôt des interprètes que des législateurs.

Lyotard, l'intellectuel postmoderne, affirme que le savoir postmoderne traverse une crise de légitimité.

On comprend à la lecture de ces énoncés pourquoi il est si facile d'attribuer aux théoriciens de la postmodernité les caractéristiques des discours qu'ils analysent. Ce retournement d'un discours sur lui-même paraît dicté par la logique, lorsque ce discours porte sur un ensemble d'expressions de la pensée auquel il appartient lui-même d'une certaine manière. Cette propension logique à réintégrer dans un métalangage le contenu des discours que vise ce métalangage a au moins deux conséquences importantes.

A. Première conséquence. Elle produit d'abord une inflation artificielle de la composante méthodologique des auteurs qui se penchent sur les productions du savoir de cette période qu'ils dénomment la postmodernité. En effet, plusieurs de ces productions étant effectivement de nature méthodologique, on prête à un commentateur de la pensée postmoderne la méthodologie qu'il décrit chez les autres. 
Par exemple, Lyotard insiste beaucoup sur les travaux du philosophe Wittgenstein, qui comportent une importante composante méthodologique. On est donc porté à gonfler la pensée de Lyotard avec les méthodes préconisées par Wittgenstein (1953).

B. Seconde conséquence. Cette conséquence ne serait pas si ruineuse si elle ne s'accompagnait d'un autre phénomène. Les pensées qui sont qualifiées de postmodernes par leurs interprètes sont non seulement variées, mais leur contenu est souvent contradictoire, en dépit du fait qu'elles partagent certains thèmes et certains traits formels. Michel Foucault et Jean Baudrillard, pour prendre un exemple extrême, appartiennent tous les deux à la "postmodernité» en ce sens irréfutable qu'ils ont produit d'importants ouvrages après 1970. On sait toutefois que Baudrillard a publié un terrible pamphlet contre la pensée de Foucault (Oublier Foucault, 1977). On ne saurait donc, sans engendrer la plus profonde des confusions, combiner ces deux pensées pour en tirer une méthodologie originale ou une façon de penser commune. Or, ces amalgames dépareillés sont d'autant plus fréquents que les théoriciens de la postmodernité accordent un privilège systématique à l'intertextualité et qu'ils tentent de s'insérer dans des traditions intellectuelles qui leur étaient initialement étrangères. Par exemple, Norris (1990: 29) procède précisément à l'amalgame très instable de la pensée de Baudrillard, de Foucault et de Lyotard.

Il est à cet égard saisissant de constater que les chercheurs français trouvent souvent les éléments d'une méthodologie postmoderne chez les Anglo-saxons, les Allemands et les Italiens (Lyotard, 1979: 20-24; Raulet, lorsqu'il tente d'expliquer à Foucault le postmoderne, voir Foucault et Raulet, 1983 : 205 ; Ruby, 1990 : 223-227); on constate en même temps que les Anglo-saxons et les Allemands privilégient la pensée française lorsqu'ils recherchent l'origine du courant postmoderne (Habermas, 1982 et 1985; Kemper, 1988 et 1990; une série d'ouvrages anglo-saxons récents citent très abondamment les travaux de Derrida et de Foucault et, dans une moindre mesure, ceux de Baudrillard et de Lyotard, Bauman, 1987 et 1991; Bernstein, 1992; Boyne et Rattansi, 1990; Callinicos, 1989; Giddens, 1990 et 1991; Harvey, 1989; Marsh et Caputo, 1992; Rotman, 1987; Rosenau, 1992; Shapiro 1992; Silverman, 1990).

Prises ensemble, les deux conséquences dont nous venons de faire état engendrent non seulement la croyance dans l'existence d'une 
méthodologie en grande partie fictive qui n'existe que par personnes interposées, mais elles confrontent le chercheur à une combinatoire de fragments d'épistémologie aussi disparates qu'ils sont nombreux. En tant que méthodologie des sciences sociales, le postmoderne ne tient aucunement dans un ensemble de règles opératoires, mais plutôt dans un programme d'innovations dont le contenu reste à être explicité.

\section{De quelques constantes de la pensée postmoderne}

Le résultat des analyses précédentes peut être ainsi résumé. La pensée postmoderne est le produit complexe d'un assemblage de pensées, dont les composantes varient d'un théoricien de la postmodernité à un autre. Ces assemblages, qui présentent tout de même certaines convergences, doivent faire l'objet d'un travail de restructuration élaboré avant de pouvoir servir de méthode et de fournir les éléments d'une nouvelle épistémologie, que nous sommes encore loin de posséder. Il n'est même pas sûr que le concept d'une épistémologie postmoderne soit viable, puisque certains théoriciens du postmodernisme annoncent précisément la fin de l'épistémologie (par exemple, Lyotard en se référant à Feyerabend, 1979; Giddens interprète le postmoderne comme une dissolution de l'épistémologie - voir Giddens, 1990: 150, les premier et huitième traits du tableau 2).

Nous avons jusqu'ici délibérément insisté sur le caractère hétérogène de la pensée postmoderne afin de dissiper certains malentendus propres à engendrer de faux espoirs de renouvellement des paradigmes dans le champ des sciences sociales. Il n'en demeure pas moins qu'on peut identifier des thèmes et des procédures de travail communs à plusieurs penseurs qualifiés de postmodernes, même s'ils répugnent eux-mêmes à accepter cette désignation. Nous allons maintenant nous pencher sur ces thèmes et sur ces procédures (voir également l'annexe de cet article). Rappelons que nos analyses porteront sur le postmoderne envisagé comme un courant de la pensée théorique et qu'à l'occasion elles porteront également sur cette période de l'histoire qualifiée de postmodernité. Nous ne traiterons pas du postmodernisme défini comme une esthétique. 


\subsection{L'origine du terme «postmoderne»}

Rose (1991) présente une analyse magistrale de l'origine des termes postindustriel et postmoderne. Nous ne retiendrons que son traitement du qualificatif postmoderne, que nous compléterons par l'article de Welsch. Werner Welsch a découvert que les premières occurrences de l'adjectif postmoderne apparaissaient dès 1870 dans l'œuvre écrite du peintre anglais John Watkins Chapman. Néanmoins, Welsch fixe la naissance du terme à 1917, dans l' œuvre de Rudolf Pannwitz, intitulée Die Krisis der europaïschen Kultur (La crise de la culture européenne). Bien qu'elle connaisse les travaux de Welsch, Rose (1991: 171 et 180) propose de fixer la première apparition du mot «postmoderne» en 1934, dans l'œuvre de Frédéric de Oniz, théoricien de la littérature. Ce dernier créa l'adjectif pour désigner la poésie espagnole et sud-américaine produite de 1905 à 1914, poésie qu'il estimait être en réaction contre les excès du modernisme. Les secondes occurrences de l'adjectif «postmoderne» répertoriées par Rose (1991 : 171-172) apparaissent à partir de 1939 dans l'œuvre de l'historien Arnold Toynbee et désignent, comme on l'a vu, une époque qui commence avec la guerre de 1914 et dont le début sera ramené par la suite, par Toynbee, à une date antérieure, soit en 1875 .

Nous nous rangeons du côté de Welsch, qui fixe la date de naissance de l'expression «postmoderne» à 1917, dans l'œuvre de Pannwitz. Le passage de La crise de la culture européenne où Pannwitz exprime ce qu'il entend par «l'homme postmoderne» se lit en effet comme suit:

L'homme postmoderne, cet être trempé dans l'acier du sport, dont la conscience est nationaliste, qui est un produit du militarisme et qui est en proie à l'exaltation religieuse, n'est en réalité qu'une bête molle recouverte d'une croûte, un moyen terme entre le décadent et le barbare, qui émerge du tourbillon engendré par la grande décadence de la révolution radicale du nihilisme européen. (Cité par Welsch, 1988: 12; c'est nous qui traduisons)

En dépit de son ton qui frôle l'hystérie, ce passage conjugue suffisamment de thèmes qui seront perçus plus tard à tort ou à raison comme caractéristiques du postmoderne - la décadence, la mollesse, le chaos et le nihilisme - pour qu'on reconnaisse chez lui le lieu de la naissance de l'adjectif postmoderne. 


\subsection{Charles Wright Mills: une vision contemporaine du postmoderne}

Il faut toutefois attribuer à C. Wright Mills l'élaboration d'une perspective sur le postmoderne qui a valeur annonciatrice des développements postérieurs. Dans une conférence présentée en janvier 1959 au London School of Economics (LSE) et reproduite dans The sociological Imagination, au chapitre 9, C. Wright Mills nous présente une démonstration probante de la puissance de sa propre imagination sociologique. Cette conférence est intitulée On freedom and reason (Sur la liberté et la raison; voir Wright Mills, 1959: 165-176).

Wright Mills (1959: 66-67) nous propose de scinder l'histoire de l'Occident en quatre grands âges, soit l'Antiquité, caractérisée par la prévalence d'influences orientales, un Âge des ténèbres qui correspond au Moyen Âge, l'Âge moderne et, finalement, une période postmoderne que Wright Mills désigne comme la Quatrième Époque. Wright Mills n'est pas très précis sur le moment où s'effectue la transition entre l'Âge moderne et la Quatrième Époque. La marque de ce passage est l'épuisement des idéaux issus du Siècle des lumières. Toutefois, Wright Mills semble voir dans le libéralisme de John Stuart Mills et dans le socialisme de Marx un prolongement et même une mise en application des idéaux $\mathrm{du}$ Siècle des lumières. Le Quatrième âge commencerait avec la mise en place de sociétés - le capitalisme tardif caractérisé par la généralisation sauvage de l'économie de marché et la constitution du Bloc communiste - qui pervertissent les réformes respectivement défendues par Stuart Mills et par Karl Marx. Dans cette optique, le Quatrième Âge, ou la postmodernité, pourrait avoir une naissance aussi tardive que le début de la Guerre froide, après la fin du second conflit mondial. Cette interprétation est confirmée par la lecture du chapitre 10 de Wright Mills (1959: 177-194), qui reproduit une conférence intitulée On Politics (Sur la politique). Cette conférence fut présentée en janvier 1959, à Varsovie, sous l'égide de l'Académie polonaise des sciences et fait suite à celle présentée à la LSE. Rose (1991: 11, 171 et 195, note 52) s'étant initialement appuyée sur le Oxford English Dictionary (OED) pour répertorier les premiers usages du mot "postmoderne», elle ne mentionne pas cette seconde conférence. Reiner (1992a) ne mentionne pas non plus le chapitre 10 de Wright Mills (1959). 
Or, non seulement ce chapitre continue-t-il à développer le concept de «postmoderne», mais il nous permet d'apporter plusieurs précisions sur l'esquisse présentée dans le chapitre 9. Une première précision concerne la période caractérisée comme postmoderne. Il semble bien qu'elle coïncide avec la fin de la Deuxième Guerre mondiale. Après avoir décrit des phénomènes de centralisation qui opèrent dans les domaines de l'économie, de la politique et du monopole de la force armée, Wright Mills écrit:

L'apogée postmoderne (souligné dans le texte) de ces trois développements pris ensemble en économie, en politique, et dans la violence, a maintenant lieu le plus dramatiquement aux États-Unis et en URSS. À notre époque, les moyens tant nationaux qu' internationaux de faire l'histoire sont en train d'être centralisés. (Wright Mills, 1959: 183; c'est nous qui traduisons)

Cette polarisation de la conjoncture internationale en deux superpuissances est typique de la Guerre froide, qui semble donc la période ouvrant véritablement la Quatrième Époque ou la période postmoderne.

Cette première question réglée, on peut s'interroger sur les caractéristiques de cette période postmoderne, telles que conçues par Wright Mills. Or, ses positions vont jusqu'à préfigurer la profonde ambivalence qui marque les théories du postmoderne. Certaines de ces théories postmodernes décrivent les modalités d'une série de crises qui frappent divers champ d'activités et sont empreintes d'un certain pessimisme quant à notre capacité de les résoudre. Cette variante pessimiste du postmoderne est peut-être celle qui regroupe le plus grand nombre de théories. Elle n'est toutefois pas la seule. Des auteurs, qui résisteraient toutefois à ce qu'on leur applique le qualificatif de postmodernes, mais qui consacrent une partie considérable de leurs travaux à définir les traits du postmoderne, professent une doctrine moins désenchantée sur nos capacités à triompher des risques indéniables qui pèsent sur notre situation présente. Parmi eux, on trouve des auteurs comme Bell (1976), Habermas, (1981a et b, 1985), Callinicos (1989) et Giddens (1990, 1991).

Or, cette oscillation entre le désenchantement et un optimisme mesuré est exemplairement présente chez Wright Mills. La conférence intitulée On reason and freedom, qui a reçu le plus d'attention de la part des commentateurs, représente le moment pessimiste de la pensée de Wright Mills. En effet, ce qui caractérise ce Quatrième Âge qui succède à la modernité est la rupture du lien constitutif de l'idée de progrès entre la rationalité et la liberté. D'où une triple crise: 
(i) Une première crise, genératrice des deux autres, est précipitée par le soupçon généralisé qu'un accroissement de la rationalité ne résulte pas nécessairement dans l'approfondissement de notre libération. Il se peut même que la raison nie la liberté.

(ii) Dissociée de ses effets bénéfiques, la raison devient contestée comme valeur. C'est alors l'ensemble des incarnations de la rationalité - la science, l'éducation, la conscience de classe et la rationalisation des institutions - qui est menacé d'entrer en crise.

(iii) La crise au niveau des expressions de la pensée se reproduit de deux façons différentes au niveau de la praxis, au sein de laquelle se réalise l'action humaine. D'abord, soupçonneuse de la raison, la pratique se déleste du standard qui lui permettait auparavant de décider du meilleur cours d'action à suivre. De façon plus profonde peut-être, c'est l'identification d'un sujet individuel libre comme agent de l'histoire qui devient problématique.

Cette conscience de crise, qui s'exprime dans le chapitre 9 de Wright Mills (1959), évolue dans le chapitre 10. Wright Mills part de l'observation faite par Marx dans Le dix-huit Brumaire selon laquelle les hommes sont les auteurs de leur propre histoire, bien qu'ils ne puissent la faire entièrement à leur gré, n'ayant pas choisi les circonstances dans lesquelles ils font cette histoire humaine. Il remarque que l'un des indices les plus révélateurs de la situation contemporaine - qu'il s'apprête à qualifier de postmoderne - est l'accroissement très considérable du pouvoir dont disposent les êtres humains pour produire leur histoire et surtout la centralisation du pouvoir de décider comment utiliser ces ressources immenses dans les mains peu nombreuses d'une élite. Ces deux facteurs amènent Wright Mills à penser que la capacité des Occidentaux d'être consciemment les agents de leur histoire est maintenant unique.

Cette situation est décrite par Wright Mills comme paradoxale: il est en effet ironique de constater quau moment même ou les êtres humains disposent de ressources pour échapper à la fatalité et être les artisans de leur destin, les idéologies qui définissent le sujet humain comme producteur de son destin et qui l'incitent à assumer ce rôle avec détermination sont en déclin et même près de l'effondrement. Derrière cet effondrement se profile une défection de la classe intellectuelle (la trahison des clercs chère à Julien Benda et qui est maintenant un thème revenant à la mode dans les travaux de Finkielkraut, 1987, et de Ferry 
et Renaut, 1987, 1985a et b). Ces travaux, comme plusieurs autres, s'efforcent de redécouvrir les valeurs défendues au Siècle des lumières par Kant, Fichte et Schelling. Dans ce qui peut maintenant apparaître comme la partie la plus utopique de son exposé, Wright Mills assigne aux praticiens des sciences sociales (social scientists) la mission de reprendre le flambeau et de revitaliser les idéaux du Siècle des lumières:

Je ne crois pas que la preuve est faite de la nécessité d'abandonner les valeurs de la liberté et de la raison, ainsi qu'elles pourraient maintenant orienter le travail des sciences sociales. (Wright Mills, 1959: 193; c'est nous qui traduisons)

Son appel est maintenant largement entendu. En effet, dans ce combat pour l'avenir qui constitue pour beaucoup la conjoncture intellectuelle présente, les deux partis qui s'affrontent sont précisément des penseurs postmodernes, qui déconstruisent les idéaux élaborés pendant la période des Lumières, et de nouveaux apôtres de la raison, qui réaffirment la pertinence des idéaux du Siècle des lumières.

\subsection{Le postmoderne: esquisse d'une thématique}

Nous allons maintenant présenter quelques thèmes qui sont élaborés par la pensée postmoderne. Qualifiant cette présentation d'esquisse, nous en reconnaissons d'emblée le caractère incomplet; nous sommes en outre conscient que certains des traits constituant notre esquisse ne manqueront pas d'apparaître contestables.

Une brève remarque d'ordre méthodologique avant de commencer. On ne devra pas se surprendre de trouver dans notre esquisse des thèmes qui proviennent de pensées très critiques envers le postmoderne, notamment celle de Habermas. Jouant systématiquement de l'intertextualité, la pensée postmoderne est remplie d'emprunts. Toutefois, deux choses la distinguent. (i) Cette pensée radicalise ses interprétations de la conjoncture présente à un point tel que l'aspect de cette conjoncture qui est ainsi interprété apparaît en rupture avec sa forme précédente, d'où l'utilisation du qualificatif de postmoderne qui consomme cette rupture. (ii) Ce privilège accordé à la discontinuité s'enracine dans une perception de l'anéantissement des Juifs d'Europe comme une catastrophe définitive quaucune tradition de rationalité ne peut assimiler et qui ne peut être pensée que comme introduisant une mutation historique. 
Ces deux traits déterminent des oppositions indépassables. Par exemple, dans ses écrits politiques, Habermas (1981 : 444-464) conçoit la modernité comme un projet inachevé (ein unvollendetes Projekt). Il s'attire cette réplique de Lyotard (1988: 36):

Mon argument est que le projet moderne (de réalisation de l'universalité) n'a pas été abandonné, oublié, mais détruit, «liquidé». Il y a plusieurs modes de destruction, plusieurs noms qui en sont les symboles. «Auschwitz» peut être pris comme un nom paradigmatique pour l'«inachèvement» tragique de la modernité.

Cette perception de la Shoab comme un désastre sans précédent, comme un avilissement sans retour de la vie, a été profondément influencé par la Dialectique négative de Theodor Adorno.

\subsubsection{La crise de légitimation}

L'ouvrage fondamental de Habermas sur la crise de légitimation dans les sociétés capitalistes avancées a été publié en 1973 en Allemagne. Sa traduction française sous le titre Raison et légitimitéa été publiée en 1978. C'est en 1979 que Jean-François Lyotard fit paraître La condition postmoderne qui était le fruit d'une commandite du Conseil québécois des universités. Le président du Conseil avait demandé à Lyotard un rapport sur l'état du savoir dans les sociétés les plus développées. En dépit de son caractère d'écrit de circonstance, explicitement indiqué par Lyotard lui-même (Lyotard, 1979: 9), cet ouvrage a connu une très grande fortune et a été considéré, selon l'expression utilisée par Welsch (1988: 36 , note 46) dans sa généalogie du postmoderne, comme une «bible».

Lyotard (1979: 17) reconnaît dans la légitimation le problème cardinal du savoir contemporain. Il affirme donner à cette notion de légitimation un sens plus étendu que celui qui lui est conféré par les théoriciens allemands, et notamment par Habermas, dont le nom est explicitement mentionné (Lyotard, 1979: 19, note 27). Les Allemands entendraient par le concept de légitimation la possession d'une justification de l'exercice de l'autorité. Lyotard définit la légitimation comme «le processus par lequel un législateur se trouve autorisé à promulguer (une) loi comme une norme» (Lyotard, 1979: 19). Cette définition est pleinement conciliable avec la perspective de Habermas, qu'elle reprend d'ailleurs en grande partie, et nous tenterons plus tard de démarquer la pensée de Lyotard de celle de Habermas d'une façon plus précise. 
Il nous importe toutefois de bien marquer la fécondité de la position du problème de la légitimation par Habermas, qui s'inscrit dans la grande tradition de la sociologie allemande. En effet, Habermas (1978: 136) pose d'abord ce problème dans le cadre du concept weberien de la «domination rationnelle», c'est-à-dire de la domination par la promulgation de règles législatives. Habermas discute ensuite d'une redoutable ambiguité dans la théorie de Max Weber. Concédant à ce dernier que tout législateur cherche à faire naître et à nourrir la croyance en sa légitimité, Habermas soulève la question suivante. Suffit-il qu'un peuple croit dans la légitimité de son législateur ou faut-il en plus qu'il ait raison de le faire? Dans le premier cas, la légitimité est un phénomène qui ne relève que de la psychologie collective et, le cas échéant, d'une théorie de la propagande. Dans le second cas, elle est justiciable d'une logique des croyances, qui s'efforce de déterminer la validité des raisons sur lesquelles elles s'appuient pour estimer qu'une autorité est légitime, et qui pose de cette façon le problème du rapport entre la vérité et la domination.

Non seulement cette position du problème nous renvoie-t-elle à des questions soulevées par Wright Mills à l'aube de la pensée postmoderne - la rationalité est-elle un facteur de libération ou d'asservissement? mais elle détermine ce qui apparaîtra de façon croissante comme l'un des enjeux majeurs de la pensée postmoderne, à savoir la détermination des rapports entre le savoir et le pouvoir. L'utilisation de ces termes connote fortement la pensée de Michel Foucault. Foucault n'est toutefois pas le seul à avoir apparié deux concepts, dont l'un relève d'une sémantique (une théorie de la signification) et l'autre d'une énergétique (une théorie de la force). Le croisement systématique de ces deux plans nous semble être une opération qui a valeur de paradigme pour le postmoderne.

\subsubsection{Réflexivité interne et réversibilité}

La logique symbolique et les travaux sur le fondement des mathématiques ont exercé l'une des influences les plus déterminantes sur la pensée de notre époque (post-1950), la figure de proue de ces recherches étant Kurt Godel qui a démontré qu'il se trouvait des énoncés sur les mathématiques qui étaient intuitivement vrais mais dont on ne pouvait fournir la preuve (sur Godel et sur la réflexivité interne, voir Hofstadter, 1979).

Sans même tenter de rendre compte des théorèmes de Godel sur la nécessaire incomplétude du savoir mathématique, nous ferons état de 
deux caractéristiques logiques des relations qui s'établissent entre des termes. Une relation est dite réflexive lorsqu'elle s'applique au terme qui en est le sujet initial. Par exemple, la relation d'équivalence peut être appliquée à son terme originel, alors qu'il n'en va pas ainsi de la relation de différence (le terme $\mathrm{A}=$ le terme $\mathrm{A}$ [il est identique ou équivalent à lui-même], alors qu'on ne peut poser sans contradiction que ce terme A est différent du terme $\mathrm{A}$ - de lui-même).

Une seconde caractéristique des relations est leur caractère symétrique ou réversible. Par exemple, A est parent de B est symétrique, B étant également parent de $A$; la relation de paternité/maternité n'est toutefois pas symétrique: si $A$ est le père ou la mère de $B$, on ne peut affirmer la réciproque, sinon par métaphore ( $B$ est le père ou la mère de A).

Ces deux caractéristiques des relations ont été élevées au rang de procédé par la pensée postmoderne, comme en témoignent les travaux de Lyotard et de Foucault. On trouve par exemple les interrogations suivantes dans La condition postmoderne, qui jouent systématiquement de la réflexivité interne de certaines relations (Lyotard, 1979: 44, 51a et b, 89):

Qu'est-ce qui prouve que ma preuve prouve?

Comment prouver la preuve?

Qui décide des conditions du vrai?

Que vaut ton argument?

Que vaut ta preuve?

Que vaut ton vaut?

C'est en première part ce recours radical à la réflexivité interne qui démarque la pensée de Lyotard de celle de Habermas. Habermas ne questionne pas la rationalité (qu'est-ce qui rend la rationalité rationnelle?), posée comme un présupposé indépassable.

La pensée de Foucault, qui répugne absolument à la désignation de postmoderne, n'en est pas moins exemplaire dans sa découverte du caractère symétrique et réversible de la relation entre savoir et pouvoir. Non seulement la croyance dans la possession d'un savoir légitime-t-elle l'exercice d'un pouvoir, mais l'exercice d'un pouvoir sur des populations captives les rend disponibles pour la constitution d'un savoir frelaté, qui les enchaîne toujours davantage (Foucault, 1975). Cette réciprocité fondamentale de la relation entre le savoir et le pouvoir est la matrice d'un effort impressionnant de recherche. 


\subsubsection{L'ordre du discours}

Nous empruntons au titre de la leçon inaugurale de Michel Foucault au Collège de France l'énoncé de ce troisième thème. Il s'agit en réalité moins d'un thème que d'un champ d'objet et des procédures méthodologiques utilisées pour le construire.

Depuis la publication au début des années 1920 des premiers travaux de Wittgenstein, l'existence d'un «tournant linguistique» dans la philosophie et les sciences sociales n'a fait que s'accentuer (Rorty, 1967). C'est peut-être le phénomème le plus caractéristique de la pensée du $\mathrm{XXI}^{\mathrm{e}}$ siècle et il a produit des effets profonds sur des théories qui auraient dû le combattre. Par exemple, le marxisme structural issu des travaux d'Althusser s'est pratiqué sinon redéfini bien davantage comme théorie des idéologies que comme une économie politique empirique.

$\mathrm{Ne}$ pouvant illustrer ce passage au métalangage en citant des auteurs parce qu'ils seraient trop nombreux, nous nous bornerons à caractériser de façon sommaire des aspects de cette évolution. Il importe de souligner que cette évolution est double. En première part, elle signifie de façon immédiate et évidente que le signe est devenu dans toutes ses manifestations - linguistique, mathématique, picturale, etc. - l'objet privilégié sinon unique de la recherche, en vertu de cette prise de position épistémologique que l'étude de la pensée humaine passe nécessairement par celle de son expression dans le signe linguistique. En outre, les méthodes utilisées dans les sciences des signes ont fait l'objet d'emprunts systématiques de la part des autres disciplines. La linguistique et la logique symbolique ont émergé comme des disciplines-pilotes, les chercheurs qui n'étudiaient pas d'emblée un langage construisant leur objet comme un système de signes fonctionnant comme une langue (on peut citer les système de parentés et les mythes étudiés par Levi-Strauss et rappeler la célèbre formule de Jacques Lacan «L'inconscient est structuré comme un langage»). Le couplage de la linguistique avec certaines variantes de la logique a donné naissance à la sémiotique, qui ambitionne d'être une théorie générale des signes. Cette remontée vers le métalangage a donc été le fruit d'une double ascension: ce qui était signe est devenu un objet de recherche prioritaire et ce qui ne l'était pas a été construit comme signe, grâce à l'usage de méthodes issues des diverses sémiotiques.

C'est dans ce primat accordé au discours que se révèle la seconde différence entre la crise de légitimation, telle que conceptualisée par 
Habermas et par les penseurs de la postmodernité. Le problème de la légitimation, avons-nous vu, est celui-là même qui est au cœur de $\mathrm{La}$ condition postmoderne (Lyotard, 1979). Or, ce problème de la légitimation n'est pas posé à propos de l'exercice de la domination politique, mais à propos de la domination épistémologique de ces législateurs/méthodologues qui prescrivent les conditions auxquelles doit satisfaire un discours pour être reconnu scientifique. En second lieu, la méthode adoptée par Lyotard pour traiter de ce problème de la légitimation des normes imposées au savoir est elle-même empruntée au plus influent des philosophes du langage - Wittgenstein - et elle se fonde sur la reconnaissance de l'hétérogénéité des «jeux de langage» (Lyotard, 1979: 19-24).

Il importe de souligner en terminant ce bref développement sur la primauté de l'ordre du discours qu'elle n'est accordée de façon également exclusive par tous les chercheurs. Des sociologues comme Anthony Giddens (1990) font porter leurs recherches sur des phénomènes économiques, politiques et sociaux qui débordent largement le cadre d'une théorie de la production scientifique et culturelle. Ce retour à des objets qui n'appartiennent pas ou très partiellement à l'ordre du discours est sensible depuis les années 1990.

\subsubsection{La différenciation}

En dépit du caractère inflationniste de la terminologie qui lui est associé et dont l'effet d'intimidation est manifeste, le postmoderne est en réalité un verrou dont la clef non seulement n'est pas cachée mais est largement divulguée. Il existe dans la littérature un large consensus pour décrire le postmoderne sous le thème de la dispersion et du vocabulaire associé à celui-ci : au premier chef, la fragmentation (Taylor, 1992), puis l'éclatement, l'émiettement, la dissémination et tous les autres termes qui signifient la séparation. Le multiculturalisme, pour donner un exemple concret, constitue un sujet de prédilection pour les postmodernes et, surtout, pour leurs critiques. Il faut se hâter de souligner l'ambivalence de cette notion de dispersion, qui possède des significations relativement antithétiques. «Disperser ses ennemis» peut signifier les mettre en fuite et donc les vaincre; pour qui en est l'objet, la dispersion est alors la manifestation d'un échec. «Se disperser dans une foule» peut par contre signifier l'infiltrer, la noyauter et éventuellement la maîtriser; dans ce cas la dispersion peut être une façon de préparer ou même d'exercer sa domination. 
Une façon plus conforme à l'histoire de la pensée de rendre compte $\mathrm{du}$ postmoderne serait d'alléguer une hypertrophie de la fonction analytique. Le mot «analyse» vient d'un terme grec qui signifie décomposition. Or, à peu d'exception près, les penseurs associés au postmoderne ont élaboré une conceptualité dont les expressions sont préfacées par le préfixe dé-, comme le terme déconstruction, légitimement associé à la pensée de Jacques Derrida, et repris par une grande partie de la critique littéraire américaine. La déconstruction est un processus qui passe un texte au crible ou, pour utiliser un vocabulaire plus contemporain, qui établit des différences qui ne sont pas pleinement maîtrisées par l'auteur du texte. Ce concept de différence est sous l'une ou l'autre de ses formes au cœur de la pensée que l'on qualifie de postmoderne et plusieurs ouvrages importants s'y réfèrent de façon explicite dans leur titre. On citera, à titre d'exemple, Différence et répétition (Deleuze, 1968), L'écriture et la différence (Derrida, 1967), Le différend (Lyotard, 1983) et Les aventures de la différence (Vattimo, 1985a; voir aussi Vattimo, 1985b).

Ce que nous avons appelé une hypertrophie de l'analyse a résulté dans autre chose que des variations sur le thème de la différence. Cette conscience hyperanalytique a donné naissance à des thèses dont l'influence est maintenant profonde.

Une première série de thèses présente une théorie innovatrice du pouvoir et sa formulation canonique se trouve dans Foucault (1975: 119-127; pour un commentaire autorisé, voir Deleuze, 1986: 77-99). Nous n'avons pas l'espace pour résumer cette théorie complexe. Il nous suffira d'y repérer la trace du thème de la dispersion dans deux affirmations concomitantes et capitales pour la pensée postmoderne : (i) «le pouvoir s'exerce à partir de points innombrables» et (ii) les rapports de pouvoir «ne peuvent exister qu'en fonction d'une multiplicité de points de résistance» (Foucault, 1976:123 et 126). La conséquence de ces deux affirmations est que le pouvoir ne se révèle qu'à celui qui pratique une approche anti-métaphysicienne et que Foucault dénommait très précisément une micro-physique du pouvoir.

Une seconde série de thèses porte sur le savoir et elle tente également de réaliser un équilibre précaire entre des affirmations en apparence conflictuelles. Une première thèse affirme que le savoir est de toutes parts investi et même assailli par le pouvoir, dont il constitue en même temps une sorte de quête. Par ailleurs, on insiste sur le fait qu'un(e) auteur(e) ne possède pas la maîtrise de son écriture et, pour reprendre la célèbre formule de Heidegger, qu'une personne est autant parlée par 
son langage qu'elle ne le parle elle-même quand elle tient un discours. Dans un texte influent, intitulé Qúest-ce qu'un auteur, Foucault mettra en cause cette notion même d'auteur. Cet assaut contre le mythe de l'auteur s'accompagne d'une offensive non moins soutenue contre la prétention du destinataire d'un message de se l'approprier sans reste. La masse des informations que nous recevons en pratiquant une multitude de jeux de langage qui ne communiquent entre eux que de manière ambiguë nous interdit d'entretenir la croyance qu'il nous est possible d'exercer une domination ordonnée sur les significations qui nous assiègent. Bien que nous ayons mentionné le nom de Foucault, ces thèses qui visent à affaiblir notre assurance de maitriser le savoir que nous produisons ou que nous recevons ne sauraient lui être entièrement ni exclusivement attribuées. On les trouve avec des accents variés chez un grand nombre des théoriciens contemporains du langage.

Qu'elles portent sur le pouvoir ou sur le langage, ces thèses affirment la nature composite, fragmentée et à certains égards insaisissable de leur objet. Il nous a paru néanmoins opportun d'utiliser le vocable différenciation pour présenter ce thème de la fragmentation. En effet, ce sont surtout les critiques de la pensée postmoderne qui utilisent le vocabulaire de la fragmentation et de la dispersion, suggérant que cette pensée se caractérise par un iconoclasme gratuit, dont les résultats sont essentiellement négatifs. Or, nous pensons que, malgré son exacerbation, la conscience analytique postmoderne nous dévoile des différences qui sont parfois décisives là où nous nous imaginions à tort que régnait l'identique. Les travaux qui portent sur la déviance et sur le crime comportent une forte propension à croire que les phénomènes qu'ils décrivent sont d'une complexité aussi pauvre que le vocabulaire descriptif utilisé pour les étiqueter.

En second lieu, il existe une interprétation temporelle de la différenciation, qui constitue un processus de transformation pouvant engendrer une véritable métamorphose. Sans prendre une position ferme sur la question de savoir si la période dans laquelle nous nous trouvons est une modernité dont tous les traits sont poussés à la limite ou une postmodernité en rupture avec l'époque moderne, nous sommes toutefois convaincu que la clef de notre avenir ne réside pas dans un retour aux principes de l'Âge des lumières. Cette nostalgie d'une raison émancipatrice et universalisante nous semble nourrir un esprit d'arrièregarde. Malgré sa grandeur, le XviII siècle demeure trop petit pour qu'on $\mathrm{y}$ fasse rentrer tout le troisième millénaire. 


\subsubsection{Le désenchantement}

Nous avons utilisé le terme de désenchantement pour caractériser un dernier aspect de la pensée postmoderne. Ce trait est d'une application difficile car il est au cœur d'une polémique très vive, où l'on reproche à la pensée postmoderne son pessimisme qui tourne parfois à un cynisme susceptible d'engendrer une démobilisation politique complète. D'où l'accusation de constituer un nouveau conservatisme, maintes fois proférée contre la pensée postmoderne.

Il n'est pas facile de débrouiller cet écheveau. Il est en première part incontestable que la pensée postmoderne ne présente pas une perspective euphorisante sur notre réalité. Il importe toutefois d'ajouter que les critiques des postmodernes sont également des critiques extrêmement virulents de notre situation actuelle, qu'ils se représentent comme une modernité pervertie. De Habermas (1985) aux «critiques de la modernité» que viennent tout juste de faire paraître Alain Touraine (1992) et Gilles Lipovetsky (1992), qui n'en est à son premier essai (voir Lipovetsky, 1983 et 1988), les critiques des postmodernes ne sont elles-mêmes pas tendres pour la modernité.

A. À cet égard, où situer la différence entre les postmodernes et leurs critiques? On dira d'abord que par opposition à Habermas, Giddens, Touraine ou Ferry et Renaut, qui sont des théoriciens avares d'effets de style, des auteurs comme Derrida, Lyotard, Baudrillard ou même Foucault sont beaucoup plus près du paradigme de l'écrivain et de l'artiste. Ils sont en conséquence plus enclins à se laisser porter par le langage et, lorsqu' ils exhalent leur amertume, ils s'autorisent des «bons mots» à rebours qui les rendent vulnérables à l'accusation de cynisme et de complaisance. Dans ses derniers ouvrages, Baudrillard (1990a et b) prête particulièrement le flanc à cette accusation. Qu'on en juge à partir de ce passage de Cool Memories II (Baudrillard, 1990a: 84):

Aujourd'hui les infections fleurissent en dehors des conditions objectives - hiver, pollution, misère. Tout le monde se contamine réciproquement en toute saison. C'est un fait social total: le consensus tellement recherché sur le plan des valeurs et de la morale est obtenu sans effort par la grâce des virus. Au lieu de la convivialité, la conviralité.

B. Un second facteur tient dans des interprétations forcées de la pensée postmoderne qui tournent parfois au procès d'intention. Lorsque Foucault a tenu le propos risqué que la résistance au pouvoir n'était pas en position d'extériorité par rapport au pouvoir (Foucault, 1976: 
125-126), il a ouvert une brèche où beaucoup de ses ennemis se sont engouffrés, l'accusant d'abolir toute rébellion possible en décrétant qu'elle n'était pas différente du pouvoir auquel elle s'opposait. Pourtant, Foucault précise de façon explicite qu'une interprétation de sa pensée qui placerait «dans» le pouvoir ce qui prétend s'opposer à lui équivaut à «méconnaître le caractère strictement relationnel des rapports de pouvoir» (Foucault, 1976: 126).

C. La différence la plus profonde tient toutefois dans l'incroyance des postmodernes - ou de ceux désignés comme tels - relativement à la possibilité de réactiver les valeurs de l'Âge des lumières. Nous avons vu que toute tentative de renouer avec les valeurs du passé venait inévitablement se briser sur l'insurmontable obstacle des camps nazis de la mort.

D. Or, il faut souligner que le climat de la polémique autour du postmodernisme s'est notablement épaissi depuis la parution en traduction française de l'ouvrage-choc de Victor Farias (1987) sur les sympathies affirmées de Heidegger pour le nazisme (Heidegger a gardé jusqu'en 1945 sa carte du parti nazi). Dans son édition allemande, cet ouvrage était préfacé par nul autre que Habermas (voir Habermas, 1989a pour la traduction anglaise de cette préface; voir Habermas, 1989b, pour la position de cet auteur). Le climat s'est encore dégradé davantage avec la découverte que Paul de Man, le disciple le plus prestigieux de Jacques Derrida aux États-Unis, avait collaboré avec les autorités allemandes lors de l'occupation de la Belgique par les nazis.

Si la controverse sur le sens de l'épisode nazi dans la carrière de Heidegger a été si profonde, c'est parce que, comme l'ont justement remarqué Ferry et Renaut, "la déconstruction heideggerienne de la modernité $[. .$.$] en est venue à fournir à une très large partie de l'intel-$ ligentsia française les fondements et le style de sa critique du monde moderne» (Ferry et Renaut, 1988: 119). Nous ne reprendrons pas les pièces de ce débat, qui continue de diviser le monde académique tant en France, aux États-Unis, en Allemagne qu'en Angleterre. Nous n'en soulignerons qu'un des aspects. À l'exception de Lyotard (1988b), la plupart des grands intellectuels français associés à la critique du modernisme, et notamment Jacques Derrida (1987) et Philippe LacoueLabarthe (1988), ont tenté de produire une apologie subtile des égarements de l'ermite de la Forêt noire. Le fond de la démonstration 
de Derrida repose sur sa constatation que, contrairement à une règle qu'il s'était donnée dans un ouvrage de 1927 (Être et Temps, la section 10), Heidegger ne met plus le mot «esprit» (Geist) entre parenthèses dans son discours du rectorat de 1933, alors qu'il avait accepté d'être le recteur nazi de l'Université de Fribourg (Heidegger n'hésite pas à utiliser le vocabulaire du spiritualisme dans son discours du rectorat, alors qu'il y répugnait auparavant). Derrida tire de cette constatation initiale - assurément rigoureuse, mais également assez ténue - une interprétation très alambiquée qui amoindrit la portée de l'engagement nazi de Heidegger et de son silence accablant - hormis une seule phrase citée par Lyotard (1988b: 137) - sur l'extermination des Juifs et des Tziganes. D'où le soupçon que la déconstruction en apparence austère et implacable de la modernité menée par Derrida et par ses émules postmodernes serait un exercice beaucoup moins intransigeant qu'il n'y paraît s'il était mesuré à l'aune de leurs contorsions pour dénazifier le bonhomme Heidegger. C'est ainsi que Lyotard écrira justement dans son livre sur Heidegger et «les Juifs» (Lyotard, 1988b: 93):

Mêmes les apologies les plus sévères, celles qui déconstruisent le texte heideggerien avec le souci d'une vraie relecture, n'échappent pas au «montage». Déconstruire est aussi «monter». Jacques Derrida, j'en suis sûr, ne dirait pas le contraire: monter par le démontage.

Ce qui est navrant dans ces tribulations tient dans le discrédit qui est jeté sur la pensée postmoderne par des protagonistes aussi médiocres qu'ils sont de mauvaise foi, et qui exploitent ce retour du refoulé pour monter des cabales (par exemple, la cabale contre l'attribution d'un doctorat honoris causa à Jacques Derrida par l'Université de Cambridge).

\section{Jalons pour une autre criminologie}

La première partie de cet article a été consacrée à montrer que le postmoderne ne constituait pas une méthode autonome et que, par certains de ses aspects, il questionnait l'idée même d'une épistémologie normative. Ce serait donc contredire notre propos du début que de conclure ce texte en proclamant les canons d'une criminologie «postmoderne». Néanmoins, il est plusieurs des thèmes abordés dans la seconde partie de ce travail qui peuvent se réverbérer sur la criminologie. C'est à cet exercice de transposition que nous voulons maintenant nous livrer. 
Il importe néanmoins de marquer d'emblée la modestie de notre propos. Ces quelques jalons d'une autre criminologie, que nous nous proposons de poser, ne doivent pas être perçus comme l'esquisse d'un programme qu'on ambitionnerait de substituer éventuellement à la criminologie, telle qu'elle se produit actuellement. Nos réflexions suggèrent des ajouts et des amendements aux procédures actuellement suivies, avec lesquelles elles sont la plupart du temps compatibles.

Nous ajouterons pour faire bonne mesure que la recherche criminologique et la pensée postmoderne sont consonantes et qu'il n'est pas besoin de forcer les concepts pour les rapprocher. On sait que la pensée de Habermas et que celle de Foucault ont déjà inspiré de nombreux travaux en criminologie et la liste des auteurs qu'ils ont influencés, de Stanley Cohen à David Garland en passant par Michèle Perrot, pour Foucault, et de Louk Hulsman à Alessandro Barratta, pour Jurgen Habermas, serait longue à établir. Toutefois, même dans ses aspects les plus formels - lorsque, par exemple, Jean-François Lyotard définit la science postmoderne comme la science des instabilités (Lyotard, 1979: 88) - la pensée postmoderne connote une théorie de la déviance et de la marginalité. Robert Reiner (1992a, 1992b) a déjà commencé à intégrer des éléments du postmoderne à ses recherche sur la police. Sur un mode moins empirique, Stanley Raffel (1992) a produit une étude du concept de la justice, entendu comme contrainte normative, dans l'œuvre de Habermas et dans celle de Lyotard.

Nos propres réflexions sont regroupées sous quatre rubriques, à savoir les procédures, les objets et les problématiques de la recherche et, finalement quelques hypothèses de travail.

\subsection{Procédures}

Sous cette rubrique, nous présentons des réflexions qu'en un autre contexte nous aurions qualifiées d'épistémologiques ou de méthodologiques, au sens le plus étendu du terme. Pour des raisons énoncées dans les parties qui précèdent, nous répugnons à utiliser cette terminologie en son sens strict. Nous avons toutefois estimé qu'il n'était pas inutile de donner au lecteur un repère du lieu de notre discours. 


\subsubsection{Une épistémologie à géométrie variable}

Même les criminologues qui s'affirment les moins constructivistes, comme Cusson (1992), ne peuvent faire l'impasse sur le fait que la déviance et, a fortiori, le crime recouvrent des comportements posés comme déviants ou criminels parce qu'ils ont fait l'objet d'un jugement de valeur. Comme il n'est rien dans le monde naturel dont on peut dire qu'il porte un jugement de valeur, si ce n'est un sujet humain - que celui-ci soit conçu comme un individu, un groupe, une collectivité ou des autorités instituées -, force est de reconnaître que la déviance et le crime sont des objets construits, en ce sens minimal qu'ils sont le corrélat d'un jugement de valeur.

À partir de cette constatation de base, les opinions diffèrent largement. Certains criminologues s'appuient sur un consensus normatif transculturel pour concevoir le crime comme un phénomène objectif et, partant, quasi-naturel, selon la bonne tradition du jusnaturalisme et de l'idéalisme kantien qui définit l'objectivité d'une catégorie par son universalité. D'autres sociologues, comme Philippe Robert, soulignent le caractère si profondément hétérogène des comportements criminalisés qu'ils n'auraient en commun que la propriété d'être saisis par la norme pénale. Dans un article récent, Pires et Digneffe (1992) tentent de réconcilier le naturalisme («paradigme du fait social») et le constructivisme («paradigme de la définition sociale») par le moyen d'une théorie du sujet en situation, où les contraintes de la situation fournissent la composante naturelle et où l'agir du sujet apporte la composante structurante.

À l'exception d'un naturalisme radical qui contesterait que la déviance et le crime sont des comportements évalués, toutes ces positions sont défendables, car elles possèdent toutes une base empirique qui fournit un stock d'exemples pour les illustrer. La difficulté réside dans le fait que ces bases empiriques sont différentes et que chaque position épistémologique prétend rendre compte de l'ensemble du cadastre des comportements qui font l'objet d'un jugement de valeur négatif. Nous ne pensons pas que ce conflit entre le naturalisme et le constructivisme, qui est aussi vieux que l'épistémologie, puisse être résolu sur le terrain des principes. Au lieu donc d'arguer de manière stérile pour l'un ou l'autre membre de l'alternative ou de tenter de les réconcilier en les conviant d'occuper un juste milieu conçu de manière si abstraite qu'il ne constitue qu'un espace fictif et a-théorique, nous présenterons des stratégies nourries par le pragmatisme (Cometti, 1992). 
Nous proposons donc d'élaborer des stratégies épistémologiques qui s'ajustent aux caractères des phénomènes visés en objet. On nous objectera que cette proposition suppose que l'épistémologie se précède en quelque sorte elle-même, puisque que les caractères du phénomène sur lesquels on se fondera pour déterminer la stratégie à adopter doivent eux-mêmes être construits. Non seulement cette objection n'est-elle pas recevable, puisqu'elle présuppose que le débat qu'on veut éviter a été résolu en faveur du constructivisme, mais ce mouvement d'anticipation de la théorie sur elle-même constitue la vie même de la pratique scientifique.

Indépendamment de toute prise de position ontologique en faveur de l'existence du mal en soi, la doctrine du droit positif a accoutumé de distinguer entre les mala in se et les mala probibita. Les premières constituent le noyau dur et récurrent des codifications pénales en vigueur dans divers États (l'homicide, le viol, le vol). Les secondes constituent un ensemble de dispositions variables d'un État à l'autre et où le caractère discrétionnaire de la volonté du législateur s'affiche davantage (toutes les prohibitions sur la consommation des diverses catégories de stupéfiants). Nous ne proposons pas de façon simpliste de pratiquer une approche objectiviste avec les «maux en soi» et une approche constructiviste avec «les maux défendus». Nous suggérons plutôt que l'on distingue au moins deux statuts qui peuvent être conférés à une position épistémologique. On pourrait d'abord conférer à une position épistémologique le statut de l'hypothèse plausible, qui fournit un présupposé opératoire permettant au chercheur de commencer le travail. On peut également lui conférer le statut de l'hypothèse critique, pour laquelle l'objet visé constitue une épreuve; au terme de cette épreuve l'hypothèse critique peut se trouver validée et l'objet est alors déconstruit ou reconceptualisé, par choc en retour; ou bien l'hypothèse critique est simplement rejetée.

Illustrons cette distinction par un exemple. Soit l'homicide, qui est le prototype d'un «mal en soi», au sens de la doctrine juridique. Une position épistémologique de type objectiviste pourrait alors fonctionner avec le statut de l'hypothèse plausible et une position constructiviste avec le statut de l'hypothèse critique. Une validation de l'hypothèse critique consisterait à tenter d'entamer la naturalité de l'homicide en procédant à un démontage de l'homicide pénal, qui en révèle la singularité. Il se pourrait toutefois que l'homicide pénal soit un objet compact qui détermine sa représentation pénale plutôt qu'il n'est construit par 
elle. Dans ce cas, on devrait conclure que l'hypothèse critique n'a pas elle-même résisté à l'épreuve des faits: le savoir acquis sur l'homicide ferait alors l'objet d'une réintégration dans le cadre raffermi de l'hypothèse plausible.

Nous pourrions fournir une illustration converse d'une autre stratégie épistémologique en prenant l'exemple de l'ivresse au volant. Dans ce cas, c'est le constructivisme qui serait posé comme hypothèse plausible et l'objectivisme comme hypothèse critique, soumise à l'épreuve des faits.

Nous nous rendons bien compte du caractère très rudimentaire de nos indications. Nous espérons qu'elles transmettent néanmoins l'essentiel de notre position, qui est que le choix d'une stratégie épistémologique relève d'une pragmatique incarnée de la recherche plutôt que d'une logique universalisante.

\subsubsection{Un volontarisme de la différence}

En contraste, peut-être, avec les suggestions précédentes, la proposition que nous souhaitons faire ici est tellement simple qu'on peut lui donner la forme d'un slogan: il faut en tout pratiquer une approche différenciée. Qu'est-ce à dire? Même si l'on a maintenant tendance à reconnaître que le crime et la déviance sont des termes dont le référent est constitué par des comportements tellement hétérogènes qu'ils ne se comparent sous aucun autre rapport que leur condamnation par au moins une source de normativité, la criminologie a néanmoins tendance, sous la pression extrêmement puissante d'une opinion publique façonnée par la presse, à regrouper des éléments qu'il est impérieux de distinguer.

Les notions de violence et de prévention du crime sont les produits immédiats de cette tendance. Assimilé à la norme juridique de «crime contre la personne», il peut sembler que le concept de violence soit moins large que celui de crime. En réalité, toutes les formes de violence n'étant pas criminalisées, ce concept déborde la notion de crime et, de la violence dans les sports aux mutilations sexuelles rituelles en passant par la cruauté mentale, on l'emploie pour désigner toutes les formes dramatiques de ce qu'on condamne. De la même façon, la notion de la prévention du crime est aussi inopérante que celle de la prévention de la maladie (de quelle maladie parle-t-on? de l'asthme, du diabète ou de la méningite?).

Les regroupements précipités de comportements dénoncés ou de mesures prophylactiques sous une même désignation doivent être dis- 
solus; les parties libérées par cette dissolution seront assignées à nouveau à des catégories assez homogènes pour autoriser une pratique déterminée, en fonction de critères explicites et transformables.

\subsubsection{Quantités numériques et quantités intensives}

Soit les constatations suivantes: (i) depuis 1990, l'homicide fait chaque année plus de victimes aux États-Unis (plus de 16 000) que ne l'a fait le terrorisme international depuis 1960 (environ 14 000); (ii) par comparaison à d'autres métiers, comme la construction, celui de policier est sécuritaire; (iii) par comparaison à d'autres sports comme la motoneige ou le ski, la boxe est une activité sécuritaire; (iv) comparée au tabagisme, la toxicomanie est, au regard du nombre de ses victimes, un problème beaucoup moins dramatique.

Au regard du livre des comptes numériques, toutes ces constations sont indiscutables. D'où vient qu'en dépit de statistiques avec lesquelles il est familier, le public continue de réagir plus fortement à la mort des policiers qu'aux accidents de travail sur les chantiers de construction? La réponse se trouve évidemment dans la signification qui est attribuée à ces événements et qui est souvent - mais pas toujours - inscrite dans le vocabulaire utilisé pour les décrire. Dans le cas des décès reliés aux conditions de travail, on qualifie d'accident la chute d'un ouvrier du haut de la structure d'acier d'un édifice en construction, alors qu'on hésite à qualifier ainsi l'homicide d'un policier, toujours conçu comme le fruit plus ou moins prémédité d'une volonté humaine (il en va de même des morts sur le ring par opposition à celles sur une piste de course).

Les événements portent des significations différentes. On résiste cependant beaucoup, nous semble-t-il, à tirer les conséquences de ce truisme. Non seulement les événements sont-ils porteurs de sens différents au regard de notre intellect, mais ils suscitent des réactions d'intensité très variable par rapport à notre affectivité. C'est pourquoi il est nécessaire de prolonger leur dénombrement quantitatif, qui a jusqu'ici fait l'objet d'un privilège scientifique injustifié, par une appréhension de leur signification conceptuelle et affective. Ce n'est qu'en considérant les objets de la criminologie comme des signes susceptibles de provoquer une réaction émotionnelle et d'être affectivement décodés, autant que d'être aplatis par un décompte, qu'on sera en mesure de comprendre pourquoi un jury est prêt à abolir les libérations conditionnelles 
pour un enfant sexuellement agressé, alors que la brutalisation des prostitué(e)s est si banalisée qu'elle ne résulte même pas dans la convocation d'un jury.

\subsection{Objets}

Les remarques que nous avons faites sur la compatibilité de nos propositions avec la criminologie actuelle prennent ici tout leur sens. Il est clair que les objets dont nous proposons l'étude plus approfondie n'épuisent pas, peu s'en faut, le champ de la criminologie mais qu'ils prennent place dans ce champ, à côté des phénomènes dont s'est toujours préoccupée la criminologie.

\subsubsection{Discours, nombres, codes}

L'investissement de la criminologie dans des études de nature métalinguistique pourrait être particulièrement productive à cause de la nature de cette discipline, qui interroge des types de signes et d'objets langagiers moins traditionnels que les textes écrits. Ces objets peuvent être regroupés en trois ensembles.

A. On retrouve effectivement dans un premier ensemble le texte de la criminologie. Il nous semble toutefois que ce texte a fait jusqu'ici l'objet d'un commentaire de texte scolaire réglé par les lois de la «revue de littérature». Ce type de commentaire a pour but de résumer un texte, dans le meilleur des cas d'une façon critique, plutôt que d'analyser comment le texte produit ses effets de sens. Par exemple, dans quelle mesure le sexisme avéré de Lombroso - la femme est un être passif également incapable de génie que de délinquance; toute sa nature se définit par une sexualité qui la confine à deux destins, celui, sublimé, de mère ou celui, dégénéré, de prostituée - a-t-il influé sur le contenu de ses thèses sur le sujet criminel? Étienne de Greeff nous révèle avoir commencé à comprendre le passage au meurtre de l'assassin en réfléchissant sur les étapes de la conversion au christianisme des populations africaines. Quelle est le sens de cette intuition initiale sur le développement subséquent de l'œuvre? À très peu d'exceptions près, toutes les études criminologiques sur la discrimination raciale dans la justice pénale sont effectuées par des Blancs. Cette situation est-elle préjudiciable aux conclusions de ces recherches? On note, par exemple, qu'au Canada, 
c'est la communauté des Blancs, qui, dans un réflexe paternaliste qui nous ramène à La Case de l'oncle Tom, s'insurge sur la collection de statistiques criminelles qui identifient la race ou la communauté ethnique de l'infracteur. Ces questions qui débordent largement le cadre traditionnel du passage en revue de la documentation devraient être soulevées de manière plus systématique dans la cadre d'une archéologie du savoir criminologique.

B. La criminologie ne produit pas que du texte. Elle produit également des séries statistiques et elle s'appuie de plus sur de telles constructions pour parvenir à ses conclusions. La meilleure recherche criminologique a depuis longtemps exercé sa vigilance méthodologique à l'égard de la signification d'un ensemble de données statistiques. Or, il est de plus en plus évident que cette vigilance ne doit pas être relâchée et qu'il serait même opportun de l'accroître. L'un des lieux communs de la sociologie de la police est d'affirmer que les corps policiers consacrent la majeure partie de leurs interventions à fournir des services plutôt qu'à lutter contre le crime. Dans un travail récent, Reiner (1992b: 32) a montré que ces études sur la répartition des interventions de la police ont été effectuées à une époque où l'intervention dans le cadre d'un incident impliquant de la violence conjugale était considérée comme une fonction de service. En outre, ce type d'intervention était le plus fréquent parmi les interventions de service. Or, l'intervention policière dans le cadre de la violence conjugale ferait aujourd'hui partie des activités de répression du crime, car ce comportement est maintenant de plus en plus criminalisé. Cette simple réinterprétation du geste de classification des interventions de la police peut remettre en cause une des thèses les plus souvent répétées sur la nature du travail policier. Il serait opportun d'explorer de manière plus méthodique l'évolution des classifications du comportement dans le domaine de la justice pénale dans le cadre de la crise de la modernité et de la genèse progressive d'une postmodernité.

C. Les études sur des textes ou la critique de validité des statistiques ne sont pas des objets neufs pour la criminologie, bien que nous recommandions qu'on les aborde de façon différente ou plus systématique. La recherche sur les normes - en particulier, celle sur les normes pénales recueillies dans des codes et des droits de la personne énoncées dans des chartes - constitue toutefois une entreprise qui a été relativement négligée par les sociologues et les criminologues depuis 
la dernière décade. Les juristes faisant assez peu de recherche non liée à la pratique du droit, l'étude des normes est un domaine d'études qui pourrait être plus actif. Plusieurs motifs rendent cette réactivation désirable. En première part, la norme de comportement est, plus encore que la règle scientifique, un objet composite qui consiste en un montage parfois extrêmement complexe de formulations linguistiques et de pouvoirs institutionnels. Elle constitue donc un précipité en puissance de toutes les questions que l'on peut soulever à propos des relations de la force et du sens. En outre, la conjoncture actuelle, qu'on la désigne comme modernité ou postmodernité, est caractérisée par une surproduction juridique et parajuridique qui est massive, incontrôlée et qui risque de produire une société du litige édifiée sur un paradoxe. Ce paradoxe naît de la composition de deux tendances en apparence contradictoires, soit d'une part la généralisation du conflit et d'autre part la multiplication des contrôles qui formalisent le conflit, qui le domestiquent et lui confèrent le statut d'une marchandise que s'échangent les praticiens $\mathrm{du}$ droit et qui rendent impossible sa résolution. En résulte une société dont le blocage mène tout droit à la glaciation et dont l'agitation multiplie les feux de paille. La dissémination complètement désordonnée des droits - tout ce qui existe peut maintenant revendiquer des droits ou s'en voir attribuer - est un facteur déterminant dans le développement d'une société du paradoxe. Comme le dit Baudrillard (1990a: 31) avec justesse, «les droits de l'homme sont une structure gonflable».

\subsubsection{Représentations}

Nous jouons délibérément de l'ambiguiité du terme «représentation» pour l'utiliser à la fois pour désigner la reproduction perceptuelle d'une réalité et pour référer à l'aspect souvent dramaturgique de cette reconstruction. Ces représentations de la réalité, par exemple, par la presse écrite, sont fréquemment de nature linguistique. Néanmoins, il nous apparaît opportun de faire une catégorie à part pour l'étude des représentations, entendues comme ces reconstructions du réel qui ne sont pas soumises aux règles du discours scientifique. Prises en ce sens, les représentations sont pour l'essentiel de deux sortes, soit la reproduction de la réalité par la presse écrite et électronique, qui s'appuient de manière systématique sur les sondages, soit également toute la produc- 
tion d'œuvres dites fictives, qui comprennent autant le roman policier, le film que la série télévisée.

Il existe certaines recherches exemplaires sur la presse, sur les sondages et sur les représentations individuelles du monde de la justice et de la déviance (Ericson, Baranek et Chan, 1987, 1989 et 1991; Brillon, Louis-Guérin et Lamarche, 1984; Robert et Faugeron, 1978; Doob et Roberts, 1983). Non seulement ne sont-elles pas nombreuses, mais elles ne nous permettent pas encore de répondre à deux questions qui constituent maintenant une manière de leitmotiv. Quelle est d'abord l'influence des médias et de l'opinion publique sur la détermination des politiques pénales? Cette question est soulevée de manière rituelle à la fin des colloques sur le sentencing sans qu'on puisse faire autrement qu'improviser des réponses intuitives ou se répandre en lamentations. La seconde question a maintenant complètement été récupérée par la démagogie: c'est celle de l'influence de la représentation de la violence sur ceux et celles qui s'y livrent. En ce domaine, nous ne possédons qu'une vaste présomption de sens commun que la représentation de la violence a des effets d'entraînement sur le comportement; on manque toutefois complètement de preuves démontrant ces effets (Milavsky, 1992).

L'importance des études sur les représentations peut être ainsi montrée. L'histoire des sciences nous révèle qu'une discipline ne se constitue comme un savoir pouvant donner lieu à des applications systématiques que lorsqu'elle réussit à rompre avec les certitudes de la vie quotidienne et les thèses du sens commun. Or, tout se passe comme si dans le domaine de la justice pénale, le monde perpétuellement reconstruit des représentations formait un horizon de lieux communs rageurs, de demivérités totalitaires et de simplifications corrosives que la réflexion ne parvenait pas à crever et à qui elle risquait, accablée sous le poids de son découragement, de conférer le statut d'une donnée naturelle ou d'une fatalité auxquelles il est illusoire de prétendre s'arracher. Il est devenu urgent de reconnaître que cet horizon en apparence indépassable n'est qu'un décor médiatique dont on peut démonter les rouages.

\subsubsection{Centres et périphéries}

La conjoncture actuelle est marquée par un affrontement, beaucoup plus sérieux qu'on ne l'avait anticipé, entre des forces centripètes de rassemblement et des forces centrifuges de diversification. Ce conflit se produit 
à l'échelle intérieure des nations, où les principes d'intégration comme l'esprit républicain en France ou le melting pot aux États-Unis, nous semblent nettement en régression sur la pression de facteurs idéologiques comme le multiculturalisme et surtout sous le choc des flux migratoires. Le conflit se produit autant en politique intérieure, où des nations soudées par un pouvoir autoritaire se mettent à craquer comme des rivières gelées sous la chaleur du printemps et où des processus d'unification dont le déploiement semblait automatique se heurtent à des nationalismes plongeant des racines beaucoup plus profondes qu'on ne l'avait prévu.

Que ces enjeux se posent au niveau de la politique intérieure ou extérieure, ils intéressent la criminologie de façon immédiate. Nous n'avons pas besoin d'insister sur le caractère crucial des relations entre les groupes minoritaires ou marginalisés et les forces de l'ordre, la presse nous rappelant quotidiennement l'acuité de ce problème. D'une façon plus fondamentale, on pourrait se demander si le conflit entre la justice pénale, dans toutes ses composantes, et le multiculturalisme, ne s'alimente pas dans un antagonisme de principe. La justice pénale et, de façon plus particulière, la police sont des forces sociales centripètes et leur fin est d'imposer le consensus et de rendre le corps social homogène. Le contrôle des «étrangers» est historiquement l'une des tâches constitutives de la police. Le multiculturalisme, et les organes qui en assurent la promotion, sont au contraire des forces centripètes, dont la fin est de préserver la diversité des groupes et des ethnies. Il n'est certes pas dit que ces deux forces vont toujours évoluer sur un parcours de collision. La transformation des antagonismes en des tensions contenues va néanmoins réclamer des investissements soutenus.

Quant à l'intégration des pays dans des unités politiques plus englobantes, elle provoquera une collaboration des polices et une mise en commun des renseignements dont elles disposent qui posent à la criminologie et aux défenseurs de la vie privée un dilemme capital: doiton s'efforcer de lutter contre la mise en réseau des banques de données ou doit-on plutôt s'efforcer de considérer celle-ci comme inévitable et de faire porter tous les efforts à assurer que les renseignements accumulées sur une personne ou sur un groupe ne comportent pas d'erreurs significatives qui pourraient s'avérer extrêmement préjudiciables aux activités de cette personne ou de ce groupe? 


\subsection{Problématiques}

Les problématiques dont nous présenterons rapidement l'articulation sont au nombre de trois.

\subsubsection{L'obligation de rendre des comptes}

L'obligation de rendre des comptes ou la reddition de comptes sont des traductions françaises de l'envahissante problématique de l'accountability. Cette problématique est dans la foulée du postmoderne à deux grands titres.

Cette obligation de transparence s'inscrit d'abord dans un mouvement de réflexivité interne, qui caractérise à tous les niveaux la conjoncture actuelle. Cette exigence a suscité un tel engouement qu'il est nécessaire de rappeler qu'elle constitue une condition sous laquelle il est souhaitable qu'un organisme opère, mais qu'elle ne saurait être en soi la fin de cet organisme. On ne crée pas un organisme de façon absurde dans le seul but qu'il rende des comptes, mais on l'oblige à satisfaire à cette obligation pour vérifier dans quelle mesure il satisfait ses finalités externes. La fin de la police est la qualité de la vie et non la transparence de cette institution.

Or, il semblerait que cette constatation incontestable soit, d'une façon pratique, remise en cause par le nombre toujours croissant des instances qui exigent des comptes de la police et du système pénal. Ce fractionnement des lignes de responsabilité en une multiplicité de points d'observation que se disputent une pléthore de contrôleurs, tant officiels qu'officieux, est la seconde façon dont la problématique de la reddition des comptes possède une interface avec les pressions de la postmodernité. Le problème que pose cette multiplication des instances qui exercent des pouvoirs d'inspection est de savoir dans quelle mesure leur action ne s'annule pas réciproquement sans même qu'il y ait besoin pour l'objet d'une inspection de jouer un inspecteur contre l'autre.

\subsubsection{Légitimation}

Comme nous l'avons souligné, la problématique de la légitimation n'est pas une invention des théoriciens qui se réclament de la pensée postmoderne. Ce serait plutôt une élaboration de ceux qui se perçoivent comme les adversaires de cette pensée et, plus notamment, de Habermas. 
Il est trois traits du concept de légitimation que nous aimerions brièvement commenter. (i) Le premier de ces traits est si intimement lié à la modernité qu'il est peut être passé inaperçu. La légitimation de l'exercice d'un pouvoir normatif est perçue comme une condition nécessaire à l'efficacité de son action. Un pouvoir sans légitimation ne peut, dira par exemple Habermas (1978: 74), provoquer la somme généralisée des motivations nécessaires à son application et à son respect. (ii) Le second de ces traits est que la légitimation d'un pouvoir doit résider à l'extérieur de lui-même, dans ses effets instrumentaux - la prévention de comportements antisociaux - ou dans ses effets symboliques - le renforcement des normes (voir Baratta, 1990). (iii) La légitimation doit par définition faire l'objet d'une adhésion consensuelle - fondée sur la raison, dira Habermas.

On pouvait prédire à partir de ces traits une crise majeure du système pénal. En effet, partons du premier postulat que la légitimation est une composante nécessaire de l'exercice de l'autorité. Or, les effets instrumentaux et symboliques du système pénal sont largement au-dessous des attentes; si on la considère dans ses résultats, l'opération de ce système manifeste une prise de parti des puissants contre les faibles, comme en témoigne l'écrasante disproportion des minorités dans la population incarcérée. Relativement inefficace et discriminatoire, il devrait s'ensuivre que le système pénal est sans légitimation et qu'il devrait donc être en crise.

Or, si l'on excepte la crise budgétaire qui sévit dans toutes les composantes de l'État, non seulement le système pénal n'est pas en crise, mais à en juger par le nombre des personnes qui sont sous son contrôle, il n'a jamais été aussi prospère, ni son opération aussi recherchée. Une problématique postmoderne de la légitimation pourrait tenir compte des développements suivants. (i) Un système peut être légitimé en dépit de son échec à produire des résultats qui lui sont extérieurs, par défaut radical d'une mesure de remplacement crédible. (ii) Un système peut fonder sa légitimation sur la crainte déraisonnable des uns envers les autres, indépendamment de ses aptitudes à résoudre de façon durable ces problèmes. En d'autres termes, la légitimation peut se nourrir du conflit et de l'insécurité qu'il engendre. (iii) La légitimation ne constitue en rien une condition généralement nécessaire pour l'efficacité d'un pouvoir; elle n'est qu'un facteur dont le caractère indispensable doit être évalué dans le cadre d'une pragmatique. 
Nous ne présentons pas comme véridique cette conception à la Rorty du problème de la légitimation (voir Bauman, 1987: 197-198 et Cometti, 1992, pour la pensée de Rorty). Cette vision pragmatique du problème de la légitimation constitue néanmoins un défi que la théorie du droit et du système pénal ne peuvent éviter de relever. Si la légitimation avait quelque rapport que ce fût avec les intérêts émancipateurs de la raison, il deviendrait incompréhensible que le pape polonais de Rome continue d'être adulé.

\subsubsection{Justice et masse}

Nous n'avons pas retenu le thème de la société de masse parmi ceux qui définissent la pensée postmoderne, bien que Baudrillard (1986) ait consacré un ouvrage éloquent à la question. Cette question est cependant trop importante pour la justice pénale pour que nous la passions sous silence.

Dans une mesure presque exclusive, la justice pénale est une justice individuelle et individualisante. L'enquête policière, qui traite d'affaires individuelles, est essentiellement une procédure dirigée contre une criminalité grave et relativement peu fréquente. Les décisions qui sont rendues par les tribunaux concernent, quant à elles, la culpabilité individuelle des contrevenants. Les garanties procédurières ont été élaborées pour protéger la clientèle individuelle de ces tribunaux, en présupposant que le volume de celle-ci ne dépasserait pas la capacité d'accueil des cours de justice. Finalement, l'idéologie que revendique la magistrature au niveau de l'imposition des sanctions est celle de l'individualisation des peines. Les milieux correctionnels chargés de l'application des peines ont également adopté avec enthousiasme cette idéologie, bien qu'ils aient su qu'elle était en pratique inapplicable.

Le phénomène le plus fondamental de la justice postmoderne est celui de la surcharge du système, qui se manifeste à tous les niveaux et qui provoque son éclatement. La police est complètement débordée par le contentieux de la petite et de la moyenne délinquance et elle ne parvient pas à contrôler les violences collectives, qu'elle provoque d'ailleurs elle-même trop souvent. Non seulement les tribunaux sont débordés par le nombre des affaires, mais la procédure s'est compliquée à un tel point que le traitement individuel des affaires occasionne des délais dont l'accumulation nécessite à intervalles réguliers la prise de mesures généralisées d'abandon des poursuites. En outre, la justice est 
mal préparée pour juger d'affaires impliquant la culpabilité générique d'une personne morale (une industrie polluante, une agence gouvernementale) et pour accueillir des recours collectifs de plus en plus nombreux. La montée des populations incarcérées, avec la détérioration correspondante dans les conditions de détention, a complètement discrédité le mouvement vers l'individualisation des peines.

Le contraste entre l'individualisme de la justice pénal et le caractère massif des problèmes qu'elle doit résoudre pourrait être longuement approfondi. Pour l'essentiel, il soulève une question: comment redéfinir une justice pénale fondée sur la notion de responsabilité individuelle, de façon qu'elle puisse faire face au caractère systémique des problèmes qui lui sont soumis et qu'elle parvienne à gérer les collectivités qui constituent sa clientèle, sans renoncer au respect des droits de la personne?

\subsection{Hypothèses de travail}

Les trois hypothèses que nous présenterons ont toutes la même forme: elles se fondent sur la transformation progressive d'un ensemble de distinctions qui caractérisaient la modernité.

\subsubsection{Une nouvelle pénologie: entre surveiller et punir}

Le taux d'incarcération aux États-Unis approche les 500 personnes par 100000 habitants; plus de 1,2 millions de personnes sont incarcérées dans ce pays. Sans enregistrer la progression spectaculaire de l'incarcération qui caractérise les États-Unis, les autres pays du monde occidental, à l'exception de l'Allemagne, connaissent tous un sérieux problème de surpopulation carcérale. La solution envisagée à ce problème tient dans ce qu'on appelle aux États-Unis les peines intermédiaires (intermediate punishments). Les peines intermédiaires visent à intensifier la surveillance d'une personne de telle sorte que sa mise sous surveillance soit vécue par elle comme une peine privative de liberté. Les formes les plus fréquemment employées de peines intermédiaires sont la probation sous surveillance intensive, une alternance entre la probation et de brèves périodes d'incarcération imposées dans des circonstances assez difficiles pour constituer une thérapie de choc et l'assignation à domicile sous surveillance électronique (Petersilia, 1988; Morris et Tonry, 1990). 
La diminution de l'incarcération au profit d'une multiplication des programmes d'observation de condamné(e)s purgeant leur sentence au sein de la collectivité n'est qu'un scénario possible parmi plusieurs autres (Gordon, 1991: 223-238). Nous faisons d'abord l'hypothèse que c'est ce scénario qui se réalisera, dans la mesure où il sera perçu comme une mesure de substitution plus économique que l'incarcération (ce qui n'est pas encore assuré; voir Mainprize, 1992). En outre, sa réalisation s'accompagnera d'un important ensemble de conséquences. En première part, l'érosion de la différence entre surveiller et punir sera interprétée par l'opinion publique comme un adoucissement des peines. Pour corriger cette perception, il est prévisible que les pouvoirs publics vont s'engager dans un rétablissement de la peine de mort. Comme, d'autre part, rien n'assure qu'un recours accru aux peines intermédiaires ne provoque une diminution sensible de l'incarcération (Canada, 1987: 402-403), on pourrait se retrouver dans la situation suivante, pour ce qui est de la justice pénale: une intensification des modes les plus oppressifs de la surveillance, le développement d'un statu quo par rapport à l'usage de l'incarcération et un recours accru à la peine de mort.

Ces prévisions ne sont pas optimistes. Elles reposent sur un double constat. Nous croyons d'abord que soumis à des restrictions budgétaires, les pouvoirs publics seront contraints de limiter l'usage de l'incarcération. Ensuite, nous pensons que dans le climat punitif qui prévaut actuellement et qui est systématiquement entretenu par la presse, les autorités publiques seront conduites à équilibrer une politique de décarcération perçue par le public comme clémente (les sanctions intermédiaires) par des mesures d'accroissement de la sévérité des peines. En d'autres termes, les autorités politiques devront contenir l'incarcération en appliquant une pression aux deux extrémités de l'échelle de sévérité des peines privatives de liberté, la surveillance se substituant à l'emprisonnement vers le bas de l'échelle et l'exécution coiffant la perte de liberté dans le haut de l'échelle. Il est évidemment impérieux de trouver les moyens de conjurer le déroulement de ce scénario.

\subsubsection{Le privé et le public: un double envahissement}

Nous n'avons pas énoncé une autre des conséquences de l'érosion de la différence entre la surveillance et le châtiment, voulant en réserver l'énoncé à l'occasion de la formulation de la seconde de nos hypothèses. L'intensification de la surveillance résultera dans une pénétration plus 
profonde de la vie privée. La montée des sanctions intermédiaires n'est pas le seul facteur en cause.

On peut encore ici distinguer un double mouvement. En première approximation, nous dirons que, dans la mesure où elle est considérée comme un ensemble de renseignements, toute notre vie privée se déverse progressivement dans le domaine du public. Cette formulation doit cependant être reprise. L'opération de facteurs comme les progrès accélérés de la technologie permettant la collecte, le stockage et l'échange de renseignements sur la vie privée et l'accroissement de la volonté d'utiliser de façon systématique cette technologie à des fins multiples n'a pas pour unique résultat un déplacement de la sphère $\mathrm{du}$ privé vers le public. Elle aboutit au développement d'un moyen terme entre ces deux sphères que l'on pourrait désigner comme la sphère du non-privé. Le non-privé diffère du privé parce qu'un nombre considérable d'observateurs peuvent y avoir accès à l'insu de la personne ou de l'institution concernées par ces renseignements et sans leur permission explicite. Elle se distingue en outre du public en ce que l'accès à ces informations est clandestin, coûteux et réservé à ceux qui peuvent payer leur droit d'entrée ou qui ont assez d'astuce pour en faire l'épargne. Les renseignements accumulés par des agences comme la compagnie Équifax sur la capacité d'une personne de payer ses dettes appartiennent à la sphère du non-privé: trop de personnes peuvent y avoir un accès facile en acquittant les droits exigés par la compagnie pour qu'on puisse qualifier ces informations de privées. Par contre, elles ne sont pas en droit offertes au grand public.

Ce premier mouvement qui résulte dans un affaiblissement de la sphère du privé se redouble d'un mouvement en direction inverse, dont c'est l'autorité publique qui ressort cette fois affaiblie. Nous nous référons maintenant au mouvement très sensible de privatisation de la sécurité et du contrôle social. Ce mouvement est bien documenté et a donné lieu à des concepts originaux comme celui de la propriété privée de masse (Shearing et Stenning, 1981 : 228-229).

Notre soupçon, qu'il reste toutefois à confirmer, est que cette double avancée du public vers le privé et du privé vers le public nous obligera à repenser complètement ces deux catégories et à leur substituer un ensemble plus complexe de concepts qui nous permettront de mieux appréhender notre réalité. 


\subsubsection{La dilution de la notion d'infraction}

Nous serons brefs sur notre troisième hypothèse pour l'avoir énoncée ailleurs (Brodeur, 1992). Le principe fondateur du sentencing est qu'on ne saurait imposer une sanction à une personne à moins qu'elle n'ait commis une infraction. Une infraction est le produit d'un comportement illégal qu'on peut dater de manière précise dans le temps. Ce comportement, par exemple une fraude, peut s'étendre sur une période relativement longue de temps; néanmoins, on peut assigner à cette opération des bornes dans le temps. Pour le dire autrement, une infraction constitue une action précise qui diffère de l'état dans lequel un sujet se trouve. Un crime est quelque chose qu'on fait et non un état dans lequel on est. Par exemple, des gens qui ont été persécutés pour ce qu'ils étaient (Juifs, musulmans, Noirs, Tziganes etc.) ont toujours été considérés comme des victimes innocentes et leurs persécuteurs comme des criminels.

Or, l'hypothèse que nous nous décidons à soumettre est qu'il est possible que la répression pénale s'exerce à nouveau contre des personnes en vertu de leur état plutôt que de leur action. Cette hypothèse pourrait être vérifiée d'au moins trois manières. D'abord, la population carcérale comporte un nombre croissant de personnes incarcérées à partir de l'état où elles se trouvent (par exemple, les sans-abris arrêtés sous une inculpation qui, comme le vagabondage, signifie plutôt un état qu'une action). Dans un second cas, on distinguera diverses populations - drogués, prostitué(e)s - dont la déviance constitue un mode de vie faisant habituellement l'objet d'une tolérance qui, néanmoins, leur est parfois arbitrairement retirée (c'est là la différence entre, par exemple, une prostituée et un pickpocket: dans les deux cas la déviance est systématique. Dans le second cas, cependant, l'individu ne bénéficie d'aucune tolérance, ce qu'il fait étant irrécupérablement nuisible). Dans un troisième cas, un diagnostic médico-pénal autorise la détention indéfinie d'une personne à qui on attribue une nature d'infracteur (le cas paradigmatique étant celui du délinquant sexuel).

Ces exemples sont tirés du domaine de la justice criminelle. On pourrait leur adjoindre d'autres cas tirés de la déviance politique (la figure du dissident), des mœurs (l'intolérance envers les fumeurs), sans parler du retour des pogroms ethniques ou religieux en Europe. 
Cette problématisation de la distinction entre ce qu'une personne fait et ce qu'elle est participe de cette dérive des catégories de pensée qui semblaient les plus acquises et qui est caractéristique de la conjoncture postmoderne.

\section{En guise de conclusion}

Nous avons tenté de déduire la dernière partie de notre texte des caractères du postmoderne, tels que nous les avons décrits dans les deux premières parties. Le dernier trait que nous avons attribué à la pensée postmoderne - son pessimisme - n'a pas de trait correspondant dans notre dernière partie. Sans suppléer à ce qui n'est pas à proprement parler une lacune, nous aimerions clore cet article par une réflexion dans l'air un peu mélancolique du temps.

L'une des distinctions théoriques les plus représentatives des premiers penseurs de la modernité porte sur la différence entre un état de nature et un état de société. À l'exception de Jean-Jacques Rousseau qui présente une vision idyllique de l'état de nature, la plupart des penseurs (Hobbes, Spinoza, Locke) conçoivent l'état de nature comme une situation de conflit perpétuel soumise à la seule loi du plus fort; l'état de société, par contre, se caractérise par l'imposition de normes qui mettent un terme à la lutte de tous contre tous et qui introduisent un ordre rationnel dans le chaos de l'état de nature.

Il nous semble que la multiplication déraisonnable des normes de toute nature, loin de resserrer l'ordre et la rationalité qui devraient prévaloir dans nos sociétés contemporaines, est en train de produire l'effet inverse et de recréer un état de nature où les droits déclarés de tous sont en lutte perpétuelle contre les privilèges acquis de tous.

Dans le domaine juridique, jamais n'a-t-on eu en Amérique du Nord autant de garanties qui assurent que le déroulement des procès soit conforme aux règles de la justice. Avec le résultat qu'il n'y a presque plus de procès, une justice parallèle où se marchandent librement les plaidoyers s'étant développée pour éviter que le système des tribunaux ne s'effondre sous le poids de ses propres règles.

Sur un mode beaucoup plus dramatique, on serait tenté de voir dans l'ancienne Yougoslavie une métaphore de notre prochain état de nature (de contre-nature). La différence entre le nettoyage ethnique yougoslave et le génocide des Juifs et des Tziganes par les Nazis tient avant tout dans le fait que le second a été perpétré dans un secret relatif, alors que 
le premier s'effectue sous la surveillance de l'ONU, de l'OTAN et de la CEE et constitue un spectacle télévisé qui le dispute aux famines en Afrique. Encore une fois, l'absurde multiplication des normes, des exhortations, des conférences et des instances de contrôle produit le même effet que l'accélération d'une roue bariolée de couleur: le blanc. Cette accumulation désordonnée ne produira que des effets inertes de blanc, en dépit de toutes les couleurs qu'on voudrait continuer d'y appliquer. C'est cette idée maîtresse de la postmodernité - l'accumulation - qu'il faut se résoudre à contester. 


\section{ANNEXE}

Hassan (1985) a produit une liste d'oppositions entre le modernisme et le postmodernisme. Cette liste a été reproduite par Harvey (1989: 43) et par Rose (1991 : 49-50). Bien qu'une liste d'oppositions ne constitue pas en soi un discours explicatif, il nous a paru utile de reproduire en traduction la liste de Ihab Hassan. En outre, lorsque cela nous paraîtra opportun, nous indiquerons à quel auteur Hassan fait implicitement référence en utilisant une paire d'oppositions.

\section{Modernisme}

Romantisme/symbolisme

Forme (conjonctive, fermée)

But (intention, objectif)

Dessein

Hiérarchie

Maîtrise/logos

Objet d'art/œuvre achevée

Distance

Création/totalisation

Synthèse

Présence

Centrage

Genre/borne

Paradigme (F. de Saussure)

Hypotaxe

Métaphore (R. Jakobson)

Sélection (R. Jakobson)

Racine/profondeur

Interprétation/lecture

Signifiant (F. de Saussure)

Lisible

Narratif

Dieu le Père

Symptôme

Génital/phallique

Paranoïa

Origine/cause

Métaphysique

Déterminisme

Transcendance

\section{Postmodernisme}

Pataphysique/dadaïsme

Antiforme (disjonctive/ouverte)

Jeu

Chance

Anarchie

Épuisement/silence

Processus/performance/happening

Participation

Décréation/déconstruction (J. Derrida)

Antithèse

Absence

Dispersion

Texte/intertexte

Syntagme (F. de Saussure)

Parataxe

Métonymie (R. Jakobson)

Combinaison (R. Jakobson)

Rhizome/surface (G. Deleuze)

Contre l'interprétation/erreur de lecture

Signifié (F. de Saussure)

Scriptible (J. Derrida)

Antinarratif

Le Saint-Esprit

Désir

Polymorphe/androgyne

Schizophrénie

Différence/différence/trace (J. Derrida)

Ironie (F. Nietzsche)

Indéterminisme

Immanence 


\section{Références}

Baratta, A. (1991). Les fonctions instrumentales et les fonctions symboliques du droit pénal. Déviance et société, $X V(1), 1-25$.

Baudrillard, J. (1977). Oublier Foucault. Paris: Galilée.

Baudrillard, J. (1981). Simulacres et simulation. Paris: Galilée.

Baudrillard, J. (1986). Masses et Postmodernité. Paris: Méridiens.

Baudrillard, J. (1990a). Cool Memories II. Paris: Galilée.

Baudrillard, J. (1990b). La transparence du mal. Paris: Galilée.

Bauman, Z. (1987). Legislators and Interpreters. On modernity, post-modernity and intellectuals. Ithaca, New York: Cornell University Press.

Bauman, Z. (1991a). Intimations of Postmodernity. New York: Routledge.

Bauman, Z. (1991b). Modernity and Ambivalence. Ithaca: Comell University Press.

Bell, D. (1976). The Cultural Contradictions of Capitalism. New York: Basis Books.

Bernauer, J., \& Rasmussen, D. (Eds.) (1991). The Final Foucault. Cambridge, Massachusetts/London, England: The MIT Press.

Bernstein, R. J. (Eds.) (1985). Habermas and Modernity. Cambridge MA: The MIT Press.

Bernstein, R. J. (1992). The New Constellation. The Ethical-Political Horizons of Modernity/Postmodernity. Cambridge, Massachusetts: The MIT Press.

Boyne, R., \& Rattansi A. (Eds.) (1990). Postmodernism and society, New York: St. Martin's Press.

Brillon, Y., Louis-Guérin, C., \& Lamarche M. C. (1984). Les attitudes du public canadien envers les politiques criminelles. Montréal: Cahiers du Centre international de criminologie comparée, Cahier 1.

Brodeur, J.-P. (1992). Cartesian Penology. The Canadian Journal of Law and Jurisprudence, 5(1), 15-41.

Callinicos, A. (1989). Against Postmodernistn. A Marxist Critique. New York: St. Martin's Press.

Canada (1987). Réformer la sentence: une approche canadienne. Rapport de la Commission canadienne sur la détermination des peines. Ottawa: Ministre des Approvisionnements et Services Canada.

Cometti, J.-P. (1992). Lire Rorry. Le pragmatisme et ses conséquences. Paris : Éditions de l'Éclat.

Cusson, M. (1992). Déviance. In R. Boudon, Traité de Sociologie (389-422). Paris: Presses Universitaires de France.

Deleuze, G. (1968). Différence et répétition. Paris: PUF.

Deleuze, G. (1986). Foucault. Paris: Éditions de Minuit.

Derrida, J. (1967). L'écriture et la différence. Paris: Seuil.

Derrida, J. (1987). De l'esprit. Paris: Galilée.

Doob, A. N., \& Roberts, J. V. (1983). Analyse de l'opinion publique sur la détermination de la peine. Ottawa: Ministère de la Justice. 
Dussault, J. (1993). La Mae West du Postmodernisme. La Presse, 16 janvier 1993.

Ericson, R. V., Baranek, P., \& Chan, J. (1987). Visualizing Deviance. A Study of News Organizations. Toronto: Toronto University Press.

Ericson, R. V., Baranek, P., \& Chan, J. (1989). Negociating Control. A Study of News Sources. Toronto: Toronto University Press.

Ericson, R. V., Baranek, P., \& Chan, J. (1991). Representing Order. Crime, Law and the News Media. Toronto: University of Toronto Press.

Farias, V. (1987). Heidegger et nazisme, traduit de l'espagnol et de l'allemand par M. Benarroch et J. B. Grasset. Lagrasse: Verdier.

Ferry, L., \& Renaut, A. (1985a). La pensée 68. Essai sur l'antibumanisme contemporain. Paris: Éditions Gallimard.

Ferry, L., \& Renaut, A. (1985b). Système et critique. Essai sur la critique de la raison dans la pensée contemporaine. Paris: Ousia.

Ferry, L., \& Renaut, A. (1987). 68.86. Itinéraires de l'individu. Paris: Éditions Gallimard.

Ferry, L., \& Renaut, A. (1988). Heidegger et les Modernes. Paris: Grasset.

Feyerabend, P.(1979). Contre la méthode. Paris: Seuil.

Finkielkraut, A. (1987). La défaite de la pensée. Paris: Éditions Gallimard.

Foucault, M. (1971). L'ordre du discours. Paris: Éditions Gallimard.

Foucault, M. (1975). Surveiller et punir. Paris: Éditions Gallimard.

Foucault, M. (1976). La volonté de savoir. Paris: Éditions Gallimard.

Foucault, M. (1980). Power/Knowledge. Selected Interviews and Other Writings 1972-1977. In C. Gordon (Ed.). New York: Pantheon Books.

Foucault, M., \& Raulet, G. (1983). On Post-Structuralism: An Interview. Telos, 55, printemps, 195-211.

Foucault, M. (1991). Remarks on Marx. Conversations with Duccio Trombadori. New York: Semiotext(e).

Giddens, A. (1990). The Consequences of Modernity. Cambridge: Polity Press.

Giddens, A. (1991). Modernity and Self-Identity. Self and Society in the Late Modern Age. California, USA: Standford University Press.

Gordon, D. R. (1991). The Justice Juggernaut. Fighting Street Crime, Controlling Citizens. New Brunswick and London: Rutgers University Press.

Gottmann, J., \& Harper R. A. (Eds.) (1990). Since Megalopolis. The Urban Writings ofJean Gottmann. Baltimore et Londres: The Johns Hopkins University Press.

Harvey, D. (1990). The Condition of Postmodernity. An Enquiry into the Origins of Cultural Change. Cambridge: MA/Oxford, UK, Blackwell.

Habermas, J. (1978). Raison et légitimité. Problèmes de légitimation dans le capitalisme avancé. Paris: Payot.

Habermas, J. (1981) Die Moderne - ein unvollendetes Projekt. In J. Habermas, (1981), Kleine Polirische Schriften, 1-IV (444-464). Frankfurt am Main: Suhrkamp Verlag.

Habermas, J. (1982). Theorie des Kommunikativen Handels. Band 2, Zur Kritik des Funktionalistischen Vernunfr. Frankfurt am Main: Suhrkamp Verlag. 
Habermas, J. (1985). Der philosophische Diskurs des Moderne. Frankfurt am Main: Suhrkamp Verlag. (Cet ouvrage a été traduit en français sous le titre Le discours philosophique de la modernité. Paris: Éditions Gallimard, 1988).

Habermas, J. (1989c). Taking Aim at the Heart of the Present: On Foucault's Lecture on Kant's What is Enlightment. In J. Habermas, The New Conservatism (173-179). Cambridge, MA: Cambridge University Press.

Habermas, J. (1989a). The New Conservatism. Cambridge, MA: The MIT Press.

Habermas, J. (1989b). Work and Weltanschauung: The Heidegger Controversy from a German Perspective. In J. Habermas, The New Conservatism (140-172). Cambridge MA: The MIT Press.

Hassan, I. (1971). The Dismemberment of Orpheus: Toward a Postmodern Literature. New York: Oxford University Press.

Hassan, I. (1975). Paracriticisms: Seven Speculations of the Time. Urbana, III: University of Illinois Press.

Hassan, I. (1985). The culture of Postmodernism. Theory, Culture and Society, 2 (3), 119-132.

Hassan, I. (1987). The postmodern Turn. Essays in Postmodern Theory and Culture. Columbus, Ohio: Ohio State University Press.

Hofstadter, D. R. (1979). Godel, Escher, Bach : an Eternal Golden Braid. New York: Basic Books Inc. Publishers.

Jameson, F. (1992). Postmodernism or the Cultural Logic of Late Capitalism. Durham: Duke University Press.

Jencks, C. (1987). Post-Modernism. The New Classicism in Art and Architecture. New York: Rizzoli International Publications Inc.

Kemper, P. (1988). «Postmoderne» oder Der Kampf ton die Zukunft. Frankfurt am Main: Fischer Taschenbuch Verlag.

Kroker, A., \& Cooke, D. (1986). The Post-modern scene: excremental culture and byper-easthetics. New York: St Martin's Press.

Kroker, A., \& Cooke, D. (1988). The Postmodern Scene (2 édition). New York:Houndsmills.

Lacoue-Labarthe, P. (1987). La fiction du politique. Paris: Christian Bourgois.

Lipovetsky, G. (1983). L'ère du vide. Paris : Éditions Gallimard.

Lipovetsky, G. (1988). L'empire de l'éphémère. Paris: Éditions Gallimard.

Lipovetsky, G. (1992). Le crépuscule du devoir. Paris : Éditions Gallimard.

Lyotard, 3.-F. (1979). La condition postmoderne. Rapport sur le savoir. Paris: Éditions de Minuit.

Lyotard, J.-F. (1980). La partie de peinture. Paris: Maryse Candala.

Lyotard, J.-F. (1982). La Pintura del Segrero Nel'Epoca Postmoderna. Baruchello, Milan: Feltrineli.

Lyotard, J.-F. (1983). Le différend. Paris: Éditions de Minuit.

Lyotard, J.-F. (1988a). Le postmoderne expliqué aux enfants. Paris: Galilée.

Lyotard, J.-F. (1988b). Heidegger et les «Juifs». Paris: Galilée. 
Marsh, J. L., \& Caputo, J. (1992). Modernity and its Discontent. New York: Fordham University Press.

Milavsky, J. R. (1992). TV and Violence. Washington, D.C.: National Institute of Justice.

Morris, C. (1990). What's Wrong with Postmodernistn. Critical Theory and the End of Philosophy. New York: Harvester, Wheatsheaf.

Morris, N., \& Tonry, M. (1990). Between Prison and Probation: Intermediate Punisbments in a Rational Sentencing System. New York: Oxford University Press.

Pannwitz, R. (1917). Die Krisis der Europaschen Kultur. Werke: Band 2, Niimburg,

Petersilia, J. (1988). Expanding Options for Criminal Sentencing. Santa Monica Ca : Rand Corporation.

Portoghesi, P. (1983). Postmodern. New York: Rizzoli International Publications Inc.

Radakrishnan, R. (1989). Post-structuralist Politics: Toward a Theory of Coalition. In D. Kellner (Ed.), Post-modenasm, Jameson, Critique (301-332). Washington, D.C: Maisonneuve Press.

Raffel, S. (1992). Habermas, Lyotard and the Concept of Justice. London: MacMillan.

Reiner, R. (1992a). Fin de Siècle Blues: Policing Postmodern Society. Law Department, London School of Economics.

Reiner., R. (1992b). Myth vs Modernity: Reality and Unreality in the English Model of Policing. Law Department, London School of Economics.

Robert, P., \& Faugeron, C. (1978). La justice et son public. Les représentations sociales du système pénal. Genève: Masson, Médecine et Hygiène.

Rorty, R. (Ed.) (1967). The linguistic Turn. Receny Essays in Philosophical Method. Chicago: University of Chicago Press.

Rose, M. A. (1991). The Post-modern and the Post-industrial. A critical analysis. New York: Cambridge University Press.

Rosenau, P. M. (1992). Post-Modernism and the Social Sciences. Insights, Inroads, and Intrusions. Princeton, New Jersey: Princeton University Press.

Rotman, B. (1987). Signifying Nothing. The Semiotics of Zero. New York: St. Martin's Press.

Ruby, C. (1990). Le champ de bataille. Post-moderne/Néo-moderne. Paris : Éditions l'Harmattan.

Shapiro, M, J. (1992). Reading the Postmodern Polity. Political Theory as Textual Practice. Minneapolis/Oxford: University of Minnesota Press.

Shearing, C., \& Stenning, P. (1981). Modern Private Security. In M. Tonry \& N. Morris, Crime and Justice: an annual Review of Research (vol. 3, 193-245). Chicago: The University of Chicago Press.

Silverman, H. S. (1990). Post-Modernism, Philosophy and the Arts. New York: Routledge.

Taylor, C. (1992). Grandeur et misère de la modernité. Montréal: Bellarmin.

Touraine, A. (1992). Critique de la modernité. Paris: Arthème Fayard. 
Vattimo, G. (1985a). Les aventures de la différence. Paris: Éditions de Minuit. Vattimo, G. (1985b). La Fin de la modernité. Paris: Seuil.

Welsch, W. (1988). Postmoderne Genealogie und Bedeutung eines umstrittenen Begriffs. In P. Kemper (Her.) (1988). «Postmoderne» oder Der Kampf um die Zuleunft (9-36). Frankfurt am Main: Fisher Taschenbuch Verlag.

Wittgenstein, L. (1953). Philosophical Investigations. New York: The MacMillan Company,

Wittgenstein, L, (1961). Tractants Logico-Philosophicus. London: Routledge and Kegan Paul.

Wright Mills, C. (1959). The Sociological Imagination. London/Oxford/New York: Oxford University Press.

ABSTRACT - This article is an attempt to investigate the various meanings of the words "postmodernity», "postmodernism» and "postmodern». It also assesses the significance of these words and of the concepts that they express for criminology. The paper is divided in three parts. The first part tries to dispell important misunderstandings that have sprung in relation to postmodernisrn. The most significant of these is the belief that there is such a thing as a postmodernist "method» in the social sciences. The second part identifies the origin of the term "postmodern» and discusses various themes which are perceived to be characteristic of postmodern thought. These themes are: the present legitimation crisis, the internal reflexivity of scientific theory, discourse analysis and meta-language, social and cultural fragmentation and historical pessimism. The last part draws the consequences of the preceding analyses for the development of criminology.

KEYWORDS - Postmodernism, method, postmodern thought, discourse analysis, metalanguage, social fragmentation.

RESUMEN - Este artículo es un intento de investigar los varios significados de las palabras "postmodernidad", "postmodernismo" y "postmoderno." También se evalúa el significado de estas palabras y de los conceptos que ello expresa para la criminología. El artículo está dividido en tres partes. La primera intenta disipar importantes malentendidos que han surgido en relación con el postmodernismo. El mayor de ellos es la creencia de que existe algo llamado postmodernismo como "método" en las ciencias sociales. La segunda parte identifica el origen del término "postmoderno" y se discuten varios temas que se perciben como característicos del pensamiento postmoderno. Éstos son: la presente crisis de legitimación, la reflexividad interna de la teoría científica, el discurso analítico y el meta-lenguaje, la fragmentación social y cultural y el pesimismo histórico. La última parte elabora sobre las consecuencias del análisis previo para el desarrollo de la criminología.

Palabras Clave - Postmodernidad, método, pensamiento postmoderno, análisis de discurso, meta-lenguaje, fragmentación social. 